\title{
PRE-MULTISYMPLECTIC CONSTRAINT ALGORITHM FOR FIELD THEORIES
}

\author{
MANUEL DE LEÓN* \\ Instituto de Matemáticas y Física Fundamental, CSIC \\ C/ Serrano 123. E-28006 Madrid. Spain \\ Jesús Marín-SOlano † \\ Departamento de Matemática Económica, Financiera y Actuarial, UB \\ Av. Diagonal 690. E-08034 Barcelona. Spain \\ Juan Carlos Marrero $\ddagger$ \\ Departamento de Matemática Fundamental, Fac. Matemáticas, U. La Laguna \\ La Laguna, Tenerife. Spain \\ Miguel C. Muñoz-Lecanda ${ }^{\S}$ NARCiso Román-Royq \\ Departamento de Matemática Aplicada IV \\ Edificio C-3, Campus Norte UPC \\ C/ Jordi Girona 1. E-08034 Barcelona. Spain
}

September 27, 2017

\begin{abstract}
We present a geometric algorithm for obtaining consistent solutions to systems of partial differential equations, mainly arising from singular covariant first-order classical field theories. This algorithm gives an intrinsic description of all the constraint submanifolds.

The field equations are stated geometrically, either representing their solutions by integrable connections or, what is equivalent, by certain kinds of integrable $m$-vector fields. First, we consider the problem of finding connections or multivector fields solutions to the field equations in a general framework: a pre-multisymplectic fibre bundle (which will be identified with the first-order jet bundle and the multimomentum bundle when Lagrangian and Hamiltonian field theories are considered). Then, the problem is stated and solved in a linear context, and a pointwise application of the results leads to the algorithm for the general case. In a second step, the integrability of the solutions is also studied.

Finally, the method is applied to Lagrangian and Hamiltonian field theories and, for the former, the problem of finding holonomic solutions is also analized.
\end{abstract}

Key words: Fibre bundles, connections, multisymplectic manifolds, constraints, field theories.

AMS s.c. (2000): 35Q99, 37J05, 53C05, 53D99, 55R10, 58A20, $70 \mathrm{~S} 05$.

PACS (1999): 02.40.Vh, 11.10.Ef.

\footnotetext{
*e-mail: mdeleon@imaff.cfmacc.csic.es

${ }^{\dagger} \mathbf{e}$-mail: jmarin@ub.edu

${ }^{\ddagger} \mathbf{e}$-mail: jcmarrer@ull.es

§e-mail: matmcml@ma4.upc.edu

`e-mail: matnrr@ma4.upc.edu
} 


\section{Contents}

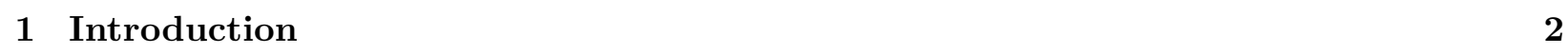

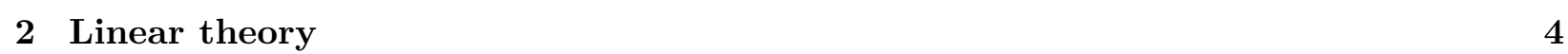

2.1 Statement of the problem. Equivalences . . . . . . . . . . . . . . . 4

2.2 Maps induced by a section $\ldots \ldots \ldots \ldots \ldots \ldots \ldots$

2.3 Characterization of solutions $\ldots \ldots \ldots \ldots \ldots$

\begin{tabular}{|llr}
\hline 3 & The general multisvmplectic case & 8
\end{tabular}

3.1 Statement of the problem . . . . . . . . . . . . . . . 8

3.2 Conditions for the existence of solutions on a submanifold of the total space . . . . . 10

3.3 The pre-multisvmplectic constraint algorithm . . . . . . . . . . . . . . . 11

3.4 The integrability algorithm . . . . . . . . . . . . . . . . 13

4 Application to Lagrangian and Hamiltonian field theories 14

4.1 Lagrangian and Hamiltonian field theories . . . . . . . . . . . . . . . . . . . . 14

4.2 Lagrangian and Hamiltonian algorithms $\ldots \ldots \ldots \ldots$

4.3 Almost-regular Lagrangians and integrable Euler-Lagrange $m$-vector fields . . . . . . 20

5 An example: affine Lagrangian densities 24

\section{Introduction}

Systems of singular differential equations have been a matter of increasing interest, especially during the last 30 years, and they have been studied separately in theoretical physics and in some technical areas such as engineering of electric networks or control theory. The fundamental characteristic of these kinds of systems is that the existence and uniqueness of solutions are not assured.

In particular, this situation arises in mechanics when dynamical systems described by singular Lagrangians are considered. Furthermore, these systems do not have a nice Hamiltonian description, since not all the momenta are available, and there is a submanifold of the momentum phase space where, in general, the dynamical equations have no solution everywhere. The same problems arise when considering systems of PDE's associated with field theories described by singular Lagrangians (indeed, many field theories are singular, for instance electromagnetism), as well as in some other applications related with optimal control theories.

Dirac [7] was pioneering in solving the problem for the Hamiltonian formalism of singular me- 
chanical systems, by developing a constraint algorithm which gives, in the favourable cases, a final constraint submanifold where admissible solutions to the dynamics exist (in the sense that the dynamical evolution remains on this manifold). Dirac's main aim was to apply this procedure to field theories. After Dirac, a lot of work was done in order to geometrize his algorithm. The first important step was the work by Gotay et al [15], and its application to the Lagrangian formalism [16. 17. Other algorithms were given later, in order to find consistent solutions of the dynamical equations in the Lagrangian formalism of singular systems (including the problem of finding holonomic solutions) 1, 24, 38, and afterwards, new geometric algorithms were developed to be applied both in the Hamiltonian and the Lagrangian formalisms [18, 20, 23, 35, 37, 42].

The Lagrangian and Hamiltonian descriptions of field theories, termed the multisymplectic approach, is the natural extension of time-dependent mechanics. Therefore, in order to understand the constraint algorithm for field theories in a covariant formalism, the first step was to develop the algorithmic procedures for time-dependent systems. This work was provided in 4, 15, 14, 19, 22, 27. 28, 29, 31, 33, 36, 43. A basic geometric study of these systems can be found in 66. Furthermore, a qualitative description of constraint algorithms for field theories was made in 9, 11.

Working within the framework of the multisymplectic description for these theories, we present in this paper a geometric algorithm for finding the maximal submanifold where there are consistent solutions to the field equations of singular theories. This algorithm gives an intrinsic description of all the constraint submanifolds. The problem is stated in a generic pre-multisymplectic fibre bundle, in order to give a solution to both Lagrangian and Hamiltonian field theories, as well as other possible kinds of systems of partial differential equations. In this framework, the solutions to these equations are given geometrically by integrable connections or, what is equivalent, by integrable locally decomposable $m$-vector fields which are transverse to the fibre projection. The key point consists in using an auxiliar connection for constructing different geometrical structures needed to develop the algorithm, by following the same methods introduced in [28] for time-dependent singular systems. This technique (the use of a connection) was used for the first time in [3], in order to obtain (global) Hamiltonian functions, and afterwards applied both in the Lagrangian and Hamiltonian formalisms for this and other purposes (see [8, 9, 14, 33, 34, 40]). An exhaustive use of this technique in mechanics and field theory can be found in [31, 32, 39].

First, the problem is reduced to another in the realm of linear algebra, and solved in this context, and then the results are applied to the general pre-multisymplectic framework. In this way, a constraint algorithm can be developed giving a sequence of submanifolds which, in the best case, ends in some final constraint submanifold where field equations have consistent solutions (connections or $m$-vector fields), although not necessarily integrable. The problem of integrability is considered and solved separately. Finally, Lagrangian and Hamiltonian field theories are particular cases where the above results are applied straightforwardly, although in the Lagrangian case the problem of finding holonomic solutions must be also analized.

The paper is organized as follows:

First, in Section 2] we state and solve the algebraic version of the problem. Then, in Section 3. we pose the general problem in the context of a pre-multisymplectic fiber bundle and, applying the results obtained in the previous Section, the solution is achieved after studying the additional problem of integrability. After this, Section 4 is devoted to giving the application to Lagrangian and Hamiltonian field theories, including the problem of finding holonomic solutions in the Lagrangian formalism. Finally, as a classical example, field theories described by affine Lagrangians are analyzed in Section 5. An Appendix about multivector fields and connections is also included, in order to make the paper more self-contained and readable. 
Manifolds are real, paracompact, connected and $C^{\infty}$. Maps are $C^{\infty}$. Sum over crossed repeated indices is understood.

\section{Linear theory}

\subsection{Statement of the problem. Equivalences}

The problem we want to solve can be first posed and solved in a linear algebraic way. In fact, let $\mathcal{W}$ and $\mathcal{E}$ be $\mathbb{R}$-vector spaces (although, instead of $\mathbb{R}$, another field of characteristic different from 2 can be used), with $\operatorname{dim} \mathcal{E}=m$, and $\operatorname{dim} \mathcal{W}=m+n$. Let $\sigma: \mathcal{W} \rightarrow \mathcal{E}$ be a surjective morphism, and denote $\mathrm{V}(\sigma)=\operatorname{ker} \sigma$, and by $\jmath: \mathrm{V}(\sigma) \hookrightarrow \mathcal{W}$ the natural injection. Consider the exact sequence

$$
0 \longrightarrow \mathrm{V}(\sigma) \stackrel{\jmath}{\longrightarrow} \mathcal{W} \stackrel{\sigma}{\longrightarrow} \mathcal{E} \longrightarrow 0
$$

Suppose that $\eta \in \Lambda^{m} \mathcal{E}^{*}$ is a volume element; denote $\omega=\sigma^{*} \eta$, and assume that a form $\Omega \in \Lambda^{m+1} \mathcal{W}^{*}$ and a subspace $\mathcal{C}$ of $\mathcal{W}$ are given. We denote this collection of data as $(\sigma ; \eta, \Omega ; \mathcal{C})$.

Next we consider the following problems in $(\sigma ; \eta, \Omega ; \mathcal{C})$ :

Statement 1 To find a m-vector $\mathcal{X} \in \Lambda^{m} \mathcal{C}$ satisfying that:
1. $\mathcal{X}$ is decomposable.
2. $i(\mathcal{X}) \omega=1$.
3. $i(\mathcal{X}) \Omega=0$.

Statement 2 To find a subspace $\mathcal{H} \subset \mathcal{C}$ satisfying that:
1. $\operatorname{dim} \mathcal{H}=\operatorname{dim} \mathcal{E}=m$.
2. $\left.\sigma\right|_{\mathcal{H}}: \mathcal{H} \rightarrow \mathcal{E}$ is an isomorphism.
3. $\left.[i(w) \Omega]\right|_{\mathcal{H}}=0, \forall w \in \mathcal{W}$.

Observe that condition 2 is equivalent to $\mathcal{W}=\mathcal{H} \oplus \mathrm{V}(\sigma)$.

Statement 3 To find a linear map $\mathbf{h}: \mathcal{E} \rightarrow \mathcal{C} \subseteq \mathcal{W}$ satisfying that:

1. $\sigma \circ \mathbf{h}=\operatorname{Id}_{\mathcal{E}} . \quad$ 2. $\left.[i(w) \Omega]\right|_{\operatorname{Im} \mathbf{h}}=0$, for every $w \in \mathcal{W}$.

Proposition 1 Statements 1, 2, and 3 are equivalent, that is, from every solution to some of these problems we can obtain a solution to the others.

( Proof ) $(1 \Longrightarrow 2) \quad$ Let $\mathcal{X} \in \Lambda^{m} \mathcal{C}$ be a solution to the problem 1 . As a consequence of the first condition, we have $\mathcal{X}=w_{1} \wedge \ldots \wedge w_{m}$, with $w_{\alpha} \in \mathcal{C}$. If $e_{\alpha}=\sigma\left(w_{\alpha}\right) \in \mathcal{E}$, for every $\alpha=1, \ldots, m$, by the second condition we have $\eta\left(e_{1}, \ldots, e_{m}\right)=1$, and hence $\left\{e_{\alpha}\right\}$ is a basis of $\mathcal{E}$.

Consider the subspace $\mathcal{H}=\left\langle w_{1}, \ldots, w_{m}\right\rangle$. We have obviously that $\operatorname{dim} \mathcal{H}=m$ and that the restriction $\left.\sigma\right|_{\mathcal{H}}: \mathcal{H} \rightarrow \mathcal{E}$ is an isomorphism. Furthermore, $[i(w) \Omega]\left(w_{1}, \ldots, w_{m}\right)=0$, for every $w \in \mathcal{W}$. Thus $\mathcal{H}$ is a solution to problem 2 .

$(2 \Longrightarrow 3) \quad$ Let $\mathcal{H}$ be a solution to problem 2. So $\left.\sigma\right|_{\mathcal{H}}$ is an isomorphism. If $\jmath_{\mathcal{H}}: \mathcal{H} \hookrightarrow \mathcal{C}$ is the natural injection, let $\mathbf{h}: \mathcal{E} \rightarrow \mathcal{C}$ be defined as $\mathbf{h}:=\jmath_{\mathcal{H}} \circ\left(\left.\sigma\right|_{\mathcal{H}}\right)^{-1}$. This map is a solution to problem 3 because the first condition holds straightforwardly and, as $\operatorname{Im} \mathbf{h}=\mathcal{H}$, the second condition holds. 
$(3 \Longrightarrow 1) \quad$ Let $\mathbf{h}$ be a solution to problem 3. If $\left\{e_{1}, \ldots, e_{m}\right\}$ is a basis of $\mathcal{E}$ satisfying that $\eta\left(e_{1}, \ldots, e_{m}\right)=1$, let $w_{\alpha}=\mathbf{h}\left(e_{\alpha}\right)$, and $\mathcal{X}=w_{1} \wedge \ldots \wedge w_{m}$. Then $\mathcal{X} \in \Lambda^{m} \mathcal{C}$ is a solution to problem 1 , because it is decomposable, and

$$
i(\mathcal{X}) \omega=\omega\left(w_{1}, \ldots, w_{m}\right)=\eta\left(e_{1}, \ldots, e_{m}\right)=1 .
$$

Furthermore, if $w \in \mathcal{W}$,

$$
[i(\mathcal{X}) \Omega](w)=\Omega\left(w_{1}, \ldots, w_{m}, w\right)=(-1)^{m}[i(w) \Omega]\left(w_{1}, \ldots, w_{m}\right)=0
$$

since $\mathbf{h}$ is a solution to problem 3 , and $w_{1}, \ldots, w_{m} \in \operatorname{Im} \mathbf{h}$.

\subsection{Maps induced by a section}

Consider the exact sequence (11), and let $\nabla: \mathcal{E} \rightarrow \mathcal{W}$ be a section of $\sigma$. Denote $\mathrm{H}(\nabla):=\operatorname{Im} \nabla$. We have the splitting

$$
\mathcal{W}=\mathrm{H}(\nabla) \oplus \mathrm{V}(\sigma)
$$

$\mathrm{H}(\nabla)$ is called the horizontal subspace of $\nabla$, and $\mathrm{V}(\sigma)$ is the vertical subspace of $\sigma$. Note that $\left.\sigma\right|_{\mathrm{H}(\nabla)}$ is an isomorphism. The above splitting induces the natural projections

$$
\sigma_{\nabla}^{H}: \mathcal{W} \rightarrow \mathrm{H}(\nabla) \subset \mathcal{W} \quad ; \quad \sigma_{\nabla}^{V}: \mathcal{W} \rightarrow \mathrm{V}(\sigma) \subset \mathcal{W}
$$

with $\sigma_{\nabla}^{H}+\sigma_{\nabla}^{V}=\mathrm{Id}_{\mathcal{W}}$; and, for every $w \in \mathcal{W}$, we write $w=w_{\nabla}^{H}+w_{\nabla}^{V}$, where $w_{\nabla}^{H} \in \mathrm{H}(\nabla)$ and $w_{\nabla}^{V} \in \mathrm{V}(\sigma)$ are called the horizontal and vertical components of $w$ induced by $\nabla$. In the same way we have the induced splitting

$$
\mathcal{W}^{*}=\mathrm{H}^{*}(\nabla) \oplus \mathrm{V}^{*}(\sigma)
$$

where $\mathrm{H}^{*}(\nabla)$ is identified with the set $\left\{\beta \in \mathcal{W}^{*} ; \beta \circ \sigma_{\nabla}^{V}=0\right\}$, and $\mathrm{V}^{*}(\sigma)$ with $\left\{\beta \in \mathcal{W}^{*} ; \beta \circ \sigma_{\nabla}^{H}=0\right\}$, in a natural way. This splitting of $\mathcal{W}^{*}$ induced by $\nabla$ gives rise to a bigradation in $\Lambda^{k} \mathcal{W}^{*}$ given by

$$
\Lambda^{k} \mathcal{W}^{*}=\bigoplus_{p, q=0, \ldots, k ; p+q=k}\left(\Lambda^{p} \mathrm{H}^{*}(\nabla) \oplus \Lambda^{q} \mathrm{~V}^{*}(\sigma)\right)
$$

Now, let $\mathcal{Z} \in \Lambda^{m} \mathcal{E}$ such that $\eta(\mathcal{Z})=1$. With this condition, $\mathcal{Z}$ is unique and decomposable, since $\operatorname{dim} \mathcal{E}=m$. Consider $\mathcal{Y}_{\eta}^{\nabla}=\Lambda^{m} \nabla(\mathcal{Z}) \in \Lambda^{m} \mathcal{W}$, which verifies the following properties:

1. $\mathcal{Y}_{\eta}^{\nabla}$ is decomposable, because if $\mathcal{Z}=e_{1} \wedge \ldots \wedge e_{m}$, then $\mathcal{Y}_{\eta}^{\nabla}=\nabla\left(e_{1}\right) \wedge \ldots \wedge \nabla\left(e_{m}\right)$.

2. $\omega\left(\mathcal{Y}_{\eta}^{\nabla}\right)=1$, since

$$
\omega\left(\mathcal{Y}_{\eta}^{\nabla}\right)=\sigma^{*} \eta\left(\Lambda^{m} \nabla(\mathcal{Z})\right)=\eta\left[\left(\Lambda^{m} \sigma \circ \Lambda^{m} \nabla\right)(\mathcal{Z})\right]=\eta\left[\Lambda^{m}(\sigma \circ \nabla)(\mathcal{Z})\right]=\eta(\mathcal{Z})=1 .
$$

$\mathcal{Y}_{\eta}^{\nabla}$ is said to be the $m$-vector associated to $\nabla$ and $\eta$, and it generates $\Lambda^{m} \mathrm{H}(\nabla)$.

The bigradation in $\Lambda^{k} \mathcal{W}^{*}$ induces a splitting of $\Omega$ as follows: $\Omega=\Omega^{(m, 1)}+\Omega^{\nabla}, \Omega^{(m, 1)}$ being a $(m+1)$-form of bidegree $(m, 1)$, and $\Omega^{\nabla}$ a $(m+1)$-form that includes the rest of components. Moreover, we have:

Proposition $2 \Omega^{(m, 1)}=\omega \wedge \gamma_{\eta}^{\nabla}$, where $\gamma_{\eta}^{\nabla}:=i\left(\mathcal{Y}_{\eta}^{\nabla}\right) \Omega$. Then $\Omega=\Omega^{\nabla}+\omega \wedge \gamma_{\eta}^{\nabla}$. 
( Proof ) As $\mathcal{Y}_{\eta}^{\nabla}$ generates $\Lambda^{m} \mathrm{H}(\nabla)$, it suffices to prove that $\Omega^{(m, 1)}$ and $\omega \wedge \gamma_{\eta}^{\nabla}$ coincide when acting on $\mathcal{Y}_{\eta}^{\nabla} \wedge v$, for every $v \in \mathrm{V}(\sigma)$. Thus, as $\gamma_{\eta}^{\nabla}$ vanishes on $\mathrm{H}(\nabla)$, we obtain

$$
\begin{aligned}
\Omega^{(m, 1)}\left(\mathcal{Y}_{\eta}^{\nabla} \wedge v\right) & \left.=\Omega\left(\mathcal{Y}_{\eta}^{\nabla} \wedge v\right)=\left[i\left(\mathcal{Y}_{\eta}^{\nabla}\right) \Omega\right)\right](v)=\gamma_{\eta}^{\nabla}(v) \\
\left(\omega \wedge \gamma_{\eta}^{\nabla}\right)\left(\mathcal{Y}_{\eta}^{\nabla} \wedge v\right) & =\omega\left(\mathcal{Y}_{\eta}^{\nabla}\right) \gamma_{\eta}^{\nabla}(v)=\gamma_{\eta}^{\nabla}(v)
\end{aligned}
$$

Finally, if $\mathbf{h}: \mathcal{E} \rightarrow \mathcal{C}$ is a linear map, $\nabla$ induces a splitting $\mathbf{h}=\mathbf{h}_{\nabla}^{H}+\mathbf{h}_{\nabla}^{V}$, where $\mathbf{h}_{\nabla}^{H}=\sigma_{\nabla}^{H} \circ \mathbf{h}$, and $\mathbf{h}_{\nabla}^{V}=\sigma_{\nabla}^{V} \circ \mathbf{h}$. Then, we introduce the map (endomorphism of $\mathcal{W}$ )

$$
\widetilde{\mathbf{h}_{\nabla}^{V}}=\mathbf{h}_{\nabla}^{V} \circ \sigma=\sigma_{\nabla}^{V} \circ \mathbf{h} \circ \sigma: \mathcal{W} \rightarrow \mathrm{V}(\sigma) \subset \mathcal{W}
$$

\subsection{Characterization of solutions}

In what follows, we assume that:

Assumption 1 The $(m+1)$-form $\Omega^{\nabla}$ is of bidegree $(m-1,2)$. Hence

$$
\Omega=\Omega^{(m, 1)}+\Omega^{(m-1,2)}
$$

This is equivalent to demanding that $i\left(v_{1}\right) i\left(v_{2}\right) i\left(v_{3}\right) \Omega=0$, for every $v_{1}, v_{2}, v_{3} \in \mathrm{V}(\sigma)$.

Note that if $U$ and $V$ are real vector spaces of finite dimension then $U^{*} \otimes V \cong\{\mathbf{h}: U \rightarrow$ $V \mid \mathbf{h}$ is linear $\}$. Thus, the auxiliar section $\nabla$ induces the $\mathbb{R}$-bilinear map

$$
\begin{aligned}
b_{\Omega}^{\nabla}: \quad \mathcal{E}^{*} \otimes \mathcal{C} & \rightarrow \quad\left(\mathcal{E}^{*} \otimes H(\nabla)\right) \times \mathrm{V}^{*}(\sigma) \\
\mathbf{h} & \left.\left.\mapsto \quad\left(\mathbf{h}_{\nabla}^{H}, i\left(i\left(\left[\widetilde{\mathbf{h}}_{\nabla}^{V}\right]^{*}\right) \mathcal{Y}_{\eta}^{\nabla}\right)\right) \Omega\right|_{\mathrm{V}(\sigma)}\right)
\end{aligned}
$$

where $\left.i\left(\widetilde{\mathbf{h}_{\nabla}^{V}}\right]^{*}\right) \mathcal{Y}_{\eta}^{\nabla}$ is the $m$-vector on $\mathcal{W}$ defined as follows: for every $\beta^{1}, \ldots, \beta^{m} \in \mathcal{W}^{*}$

$$
\left.\left(i\left(\widetilde{\mathbf{h}_{\nabla}^{V}}\right]^{*}\right) \mathcal{Y}_{\eta}^{\nabla}\right)\left(\beta^{1}, \ldots, \beta^{m}\right):=\sum_{\alpha=1}^{m} \mathcal{Y}_{\eta}^{\nabla}\left(\beta^{1}, \ldots,\left[\widetilde{\mathbf{h}_{\nabla}^{V}}\right]^{t}\left(\beta^{\alpha}\right), \ldots, \beta^{m}\right)
$$

Observe that, if $\mathcal{Y}_{\eta}^{\nabla}=w_{1} \wedge \ldots \wedge w_{m}$, with $w_{\alpha} \in \mathcal{W}$, then

$$
\left.i\left(\widetilde{\mathbf{h}_{\nabla}^{V}}\right]^{*}\right) \mathcal{Y}_{\eta}^{\nabla}=\sum_{\alpha=1}^{m} w_{1} \wedge \ldots \wedge \widetilde{\mathbf{h}_{\nabla}^{V}}\left(w_{\alpha}\right) \wedge \ldots \wedge w_{m}
$$

Theorem 1 The necessary and sufficient condition for a linear map $\mathbf{h}: \mathcal{E} \rightarrow \mathcal{C}$ to be a solution to the problem posed in Statement 3 is that

$$
b_{\Omega}^{\nabla}(\mathbf{h})=\left(\jmath_{\mathrm{H}(\nabla)} \circ\left(\left.\sigma\right|_{\mathrm{H}(\nabla)}\right)^{-1},-\left.\left(\gamma_{\eta}^{\nabla}\right)\right|_{V(\sigma)}\right)
$$

where $\jmath_{\mathrm{H}(\nabla)}: \mathrm{H}(\nabla) \rightarrow \mathcal{W}$ denotes the natural injection, and $\jmath_{\mathrm{H}(\nabla)} \circ\left(\left.\sigma\right|_{\mathrm{H}(\nabla)}\right)^{-1}$ is the horizontal lift associated with $\nabla$. 
( Proof $)(\Longrightarrow)$ Suppose that the linear map $\mathbf{h}: \mathcal{E} \rightarrow \mathcal{C}$ is a solution to the problem posed in Statement 3. Consider the linear map $\varphi: \mathcal{E} \rightarrow \mathcal{W}$ defined by

$$
\varphi:=\mathbf{h}-\jmath_{\mathrm{H}(\nabla)} \circ\left(\left.\sigma\right|_{\mathrm{H}(\nabla)}\right)^{-1}: \mathcal{E} \rightarrow \mathcal{W} .
$$

We have that

$$
\sigma \circ \varphi=\sigma \circ \mathbf{h}-\sigma \circ \jmath_{\mathrm{H}(\nabla)} \circ\left(\left.\sigma\right|_{\mathrm{H}(\nabla)}\right)^{-1}=\mathrm{Id}-\mathrm{Id}=0
$$

and therefore,

$$
\mathbf{h}_{\nabla}^{H}=\jmath_{\mathrm{H}(\nabla)} \circ\left(\left.\sigma\right|_{\mathrm{H}(\nabla)}\right)^{-1}, \quad \mathbf{h}_{\nabla}^{V}=\varphi
$$

Now, suppose that $e_{1}, \ldots, e_{m} \in \mathcal{E}$ such that $\eta\left(e_{1}, \ldots, e_{m}\right)=1$, and let $w_{\alpha}=\nabla\left(e_{\alpha}\right)$, for $\alpha=$ $1, \ldots, m$; thus $\mathcal{Y}_{\eta}^{\nabla}=w_{1} \wedge \ldots \wedge w_{m}$. We obtain that

$$
\mathbf{h}\left(e_{\alpha}\right)=\mathbf{h}_{\nabla}^{H}\left(e_{\alpha}\right)+\mathbf{h}_{\nabla}^{V}\left(e_{\alpha}\right)=w_{\alpha}+\mathbf{h}_{\nabla}^{V}\left(e_{\alpha}\right)
$$

As $\mathbf{h}$ is a solution to the problem, using the splitting (2), for every $v \in \mathcal{W}$, we have

$$
\begin{aligned}
0 & =\Omega\left(\mathbf{h}\left(e_{1}\right), \ldots, \mathbf{h}\left(e_{m}\right), v\right)=\Omega\left(w_{1}+\mathbf{h}_{\nabla}^{V}\left(e_{1}\right), \ldots, w_{m}+\mathbf{h}_{\nabla}^{V}\left(e_{m}\right), v\right) \\
& =\Omega^{(m, 1)}\left(w_{1}, \ldots, w_{m}, v\right)+\sum_{\alpha=1}^{m} \Omega^{(m-1,2)}\left(w_{1}, \ldots, \mathbf{h}_{\nabla}^{V}\left(e_{\alpha}\right), \ldots, w_{m}, v\right) \\
& \left.=\gamma_{\eta}^{\nabla}(v)+\left(i\left(i\left(\widetilde{\mathbf{h}_{\nabla}^{V}}\right]^{*}\right) \mathcal{Y}_{\eta}^{\nabla}\right) \Omega\right)(v)
\end{aligned}
$$

and the result follows.

$(\Longleftarrow)$ Suppose that there exists a linear map $\mathbf{h}: \mathcal{E} \rightarrow \mathcal{C}$ such that (44) holds; that is,

$$
\begin{aligned}
\mathbf{h}_{\nabla}^{H}=\sigma_{\nabla}^{H} \circ \mathbf{h} & =\jmath_{\mathrm{H}(\nabla)} \circ\left(\left.\sigma\right|_{\mathrm{H}(\nabla)}\right)^{-1}, \\
\left.\left(i\left(i\left(\widetilde{\mathbf{h}_{\nabla}^{V}}\right]^{*}\right) \mathcal{Y}_{\eta}^{\nabla}\right) \Omega\right)\left.\right|_{\mathrm{V}(\sigma)}=-\left.\gamma_{\eta}^{\nabla}\right|_{\mathrm{V}(\sigma)} & =-\left.i\left(\mathcal{Y}_{\eta}^{\nabla}\right) \Omega\right|_{V(\sigma)} .
\end{aligned}
$$

First we prove that $\mathbf{h}$ is a section of $\sigma$. In fact,

$$
\sigma \circ \mathbf{h}=\sigma \circ\left(\mathbf{h}_{\nabla}^{H}+\mathbf{h}_{\nabla}^{V}\right)=\sigma \circ \mathbf{h}_{\nabla}^{H}=\sigma \circ \jmath_{\mathrm{H}(\nabla)} \circ\left(\left.\sigma\right|_{\mathrm{H}(\nabla)}\right)^{-1}=\operatorname{Id}_{\mathcal{E}} .
$$

Furthermore, let $e_{1}, \ldots, e_{m} \in \mathcal{E}$, with $\eta\left(e_{1}, \ldots, e_{m}\right)=1$, and let $w_{\alpha}=\nabla\left(e_{\alpha}\right)$, for $\alpha=1, \ldots, m$. We have $\mathcal{Y}_{\eta}^{\nabla}=w_{1} \wedge \ldots \wedge w_{m}$. Now

$$
\mathbf{h}\left(e_{\alpha}\right)=\left(\jmath_{\mathrm{H}(\nabla)} \circ\left(\left.\sigma\right|_{\mathrm{H}(\nabla)}\right)^{-1}\right)\left(e_{\alpha}\right)+\mathbf{h}_{\nabla}^{V}\left(e_{\alpha}\right)=w_{\alpha}+\mathbf{h}_{\nabla}^{V}\left(e_{\alpha}\right)
$$

and we must prove that, if $w \in \mathcal{W}$, then $\Omega\left(\mathbf{h}\left(e_{1}\right), \ldots, \mathbf{h}\left(e_{m}\right), w\right)=0$. Note that, as $\mathbf{h}$ is a section of $\sigma$, it induces a splitting $\mathcal{W}=\mathbf{h}(\mathcal{E}) \oplus \mathrm{V}(\sigma)$, and hence $w=w_{\mathbf{h}}^{H}+w_{\mathbf{h}}^{V}$, where $w_{\mathbf{h}}^{H} \in \operatorname{Im} \mathbf{h}$ and $w_{\mathbf{h}}^{V} \in V(\sigma)$. Then

$$
\Omega\left(\mathbf{h}\left(e_{1}\right), \ldots, \mathbf{h}\left(e_{m}\right), w_{\mathbf{h}}^{H}\right)=0,
$$

and it suffices to prove that $\Omega\left(\mathbf{h}\left(e_{1}\right), \ldots, \mathbf{h}\left(e_{m}\right), v\right)=0$, for every $v \in \mathrm{V}(\sigma)$. In fact,

$$
\begin{aligned}
\Omega\left(\mathbf{h}\left(e_{1}\right), \ldots, \mathbf{h}\left(e_{m}\right), v\right) & =\Omega\left(w_{1}+\mathbf{h}_{\nabla}^{V}\left(e_{1}\right), \ldots, w_{m}+\mathbf{h}_{\nabla}^{V}\left(e_{m}\right), v\right) \\
& =\Omega^{(m, 1)}\left(w_{1}, \ldots, w_{m}, v\right)+\sum_{\alpha=1}^{m} \Omega^{(m-1,2)}\left(w_{1}, \ldots, \mathbf{h}_{\nabla}^{V}\left(e_{\alpha}\right), \ldots, w_{m}, v\right) \\
& =\left(i\left(\mathcal{Y}_{\eta}^{\nabla}\right) \Omega\right)(v)+\sum_{\alpha=1}^{m} \Omega^{(m-1,2)}\left(w_{1}, \ldots, \widetilde{\mathbf{h}_{\nabla}^{V}}\left(w_{\alpha}\right), \ldots, w_{m}, v\right) \\
& \left.=\gamma_{\eta}^{\nabla}(v)+\left(i\left(i\left(\widetilde{\mathbf{h}_{\nabla}^{V}}\right]^{*}\right) \mathcal{Y}_{\eta}^{\nabla}\right) \Omega\right)(v)=0
\end{aligned}
$$

Now, from Theorem [1 we deduce that: 
Corollary 1 A linear map $\mathbf{h}: \mathcal{E} \rightarrow \mathcal{C}$ is a solution to the problem posed in Statement 3 if, and only if

$$
\left.\mathbf{h}_{\nabla}^{H}=\jmath_{\mathrm{H}(\nabla)} \circ\left(\left.\sigma\right|_{\mathrm{H}(\nabla)}\right)^{-1} \quad, \quad\left[i\left(i\left(\widetilde{\mathbf{h}_{\nabla}^{V}}\right]^{t}\right) \mathcal{Y}_{\eta}^{\nabla}\right) \Omega\right]\left.\right|_{\mathrm{V}(\sigma)}=-\left.\gamma_{\eta}^{\nabla}\right|_{\mathrm{V}(\sigma)}
$$

Let $\mathrm{V}(\sigma)^{0} \subseteq \mathcal{W}^{*}$ be the annihilator of $\mathrm{V}(\sigma)$. It is clear that the vector spaces $\mathrm{H}^{*}(\nabla)$ and $\mathrm{V}(\sigma)^{0}$ are isomorphic. The orthogonal complement of $\mathcal{C}$ with respect to $\Omega$ and $\nabla$ is the subspace $\left(\mathcal{C}^{\perp}\right)_{\Omega}^{\nabla}$ of $\left(\mathcal{E} \otimes \mathrm{V}(\sigma)^{0}\right) \times \mathrm{V}(\sigma)$ defined by

$$
\left(\mathcal{C}^{\perp}\right)_{\Omega}^{\nabla}:=\left(\operatorname{Im} b_{\Omega}^{\nabla}\right)^{0}
$$

Then, from Theorem 11, we obtain:

Theorem 2 There exists a solution to the problem posed in Statement 3 if, and only if,

$$
\mathbf{h}^{*}\left(\jmath_{\mathrm{H}(\nabla)} \circ\left(\left.\sigma\right|_{\mathrm{H}(\nabla)}\right)^{-1}\right)-\gamma_{\eta}^{\nabla}(Z)=0 \quad, \quad \text { for every }\left(\mathbf{h}^{*}, Z\right) \in\left(\mathcal{C}^{\perp}\right)_{\Omega}^{\nabla} .
$$

Note that, if $\mathcal{C}=\mathcal{W}$ and $\left(\mathcal{W}^{\perp}\right)_{\Omega}^{\nabla}=\{0\}$, then it is clear that (66) holds. This is the case in the following Proposition:

Proposition 3 If the $(m+1)$-form $\Omega^{\nabla} \in \Lambda^{m+1} \mathcal{W}^{*}$ given by

$$
\Omega^{\nabla}=\Omega-\omega \wedge \gamma_{\eta}^{\nabla}
$$

is 1-nondegenerate (that is, the map $b_{\Omega^{\nabla}}: \mathcal{W} \rightarrow \Lambda^{m} \mathcal{W}^{*}$, defined by $b_{\Omega^{\nabla}}(v)=i(v) \Omega^{\nabla}$, for every $v \in \mathcal{W}$, is injective), then $\left(\mathcal{W}^{\perp}\right)_{\Omega}^{\nabla}=\{0\}$.

( Proof ) Let $\left(\mathbf{h}^{*}, Z\right) \in\left(\mathcal{W}^{\perp}\right)_{\Omega}^{\nabla}$. From the definitions of $b_{\Omega}^{\nabla}$ and $\left(\mathcal{W}^{\perp}\right)_{\Omega}^{\nabla}$ (eqs. (3) and (5)), we obtain that

$$
\left.\mathbf{h}^{*}\left(\mathbf{h}_{\nabla}^{\prime H}\right)+i(Z) i\left(i\left(\widetilde{\mathbf{h}_{\nabla}^{\prime V}}\right]^{*}\right) \mathcal{Y}_{\eta}^{\nabla}\right) \Omega=0
$$

for every $\mathbf{h}^{\prime} \in \operatorname{Lin}(\mathcal{E}, \mathcal{W})$. In particular, this implies that $\mathbf{h}^{*}\left(\mathbf{h}^{\prime}\right)=0$, for every $\mathbf{h}^{\prime} \in \mathcal{E}^{*} \otimes H(\nabla)$, and hence $\mathbf{h}^{*}=0$. Therefore, using (표), we deduce that

$$
\left.i(Z) i\left(i\left(\widetilde{\mathbf{h}_{\nabla}^{V}}\right]^{*}\right) \mathcal{Y}_{\eta}^{\nabla}\right) \Omega=0, \text { for every } \mathbf{h} \in \mathcal{E}^{*} \otimes \mathcal{W}
$$

As a consequence, from (17) and from assumption 1, it follows that $i(Z) \Omega^{\nabla}=0$ and, since $\Omega^{\nabla}$ is 1-nondegenerate, we have that $Z=0$.

\section{The general multisymplectic case}

\subsection{Statement of the problem}

The problem we wish to solve arises from the Lagrangian and Hamiltonian formalisms in field theories, although other kinds of systems can also be stated in this way.

The general geometrical setting for these kinds of systems consists in giving a fibred manifold $\kappa: F \rightarrow M$ (which in what follows is assumed to be a fibre bundle), where $\operatorname{dim} M=m>1$ and $\operatorname{dim} F=n+m$, and $M$ is an orientable manifold with volume form $\eta \in \Omega^{m}(M)$. We denote 
$\omega=\kappa^{*} \eta$. We write $\left(U ; x^{\mu}, y^{j}\right), \mu=1, \ldots, m, j=1, \ldots, n$, for local charts of coordinates in $F$ adapted to the fibred structure, and such that $\omega=\mathrm{d} x^{1} \wedge \ldots \wedge \mathrm{d} x^{m} \equiv \mathrm{d}^{m} x$. Let $\Omega \in \Omega^{m+1}(F)$ be a closed form, and consider the triad $(F, \Omega, \omega)$. The form $\Omega$ is said to be a multisymplectic form if it is 1-nondegenerate, that is, if the map $b_{\Omega}: \mathrm{T} F \longrightarrow \Lambda^{m} \mathrm{~T}^{*} F$, defined by $b_{\Omega}(v)=i(v) \Omega$, for every $v \in \mathcal{W}$, is injective. In this case, the system described by the above triad is called a multisymplectic system. Otherwise, the form is said to be a pre-multisymplectic form, and the system is pre-multisymplectic.

The problem is stated as follows:

Statement 4 Given a pre-multisymplectic system $(F, \Omega, \omega)$, we want to find a submanifold $\jmath_{C}: C \hookrightarrow$ $F$, and a $\kappa$-transverse, locally decomposable and integrable $m$-vector field $\mathcal{X}_{C}$ along $C$, in the fibration $\kappa: F \rightarrow M$, such that

$$
i\left(\mathcal{X}_{C}(y)\right) \Omega(y)=0 \quad, \quad \text { for every } y \in C \text {. }
$$

First we obviate the integrability condition. Hence the problem consists in finding a submanifold $C \hookrightarrow F$ and a locally decomposable $m$-vector field $\mathcal{X}_{C} \in \mathfrak{X}^{m}(F)$ along $C$ such that

$$
i\left(\mathcal{X}_{C}(y)\right) \omega(y)=1 \quad, \quad i\left(\mathcal{X}_{C}(y)\right) \Omega(y)=0 \quad, \quad \text { for every } y \in C .
$$

(Note that the first equation implies that $\mathcal{X}_{C}$ is $\kappa$-transverse).

Taking into account Remark [5] in the Appendix and Proposition [1, we have:

Proposition 4 If $C$ is a submanifold of $F$, then there exists a solution to the problem stated in Statement 4 if, and only if, at every point $y \in C$, there is $\mathbf{h}_{y} \in \mathrm{T}_{\kappa(y)}^{*} M \otimes \mathrm{T}_{y} C \cong \operatorname{Lin}\left(\mathrm{T}_{\kappa(y)} M, T_{y} C\right)$ such that

1. $\mathbf{h}_{y}$ is $\kappa$-transverse (that is, it is a connection along $C$ ):

$$
\left.\mathrm{T}_{y} \kappa\right|_{\mathrm{T}_{y} C} \circ \mathbf{h}_{y}=I d .
$$

2. For every $\left(X_{1}^{\prime}\right)_{\kappa(y)}, \ldots,\left(X_{m}^{\prime}\right)_{\kappa(y)} \in \mathrm{T}_{\kappa(y)} M$, and $Y_{y} \in \mathrm{T}_{y} F$,

$$
\Omega(y)\left(\mathbf{h}_{y}\left(\left(X_{1}^{\prime}\right)_{\kappa(y)}\right), \ldots, \mathbf{h}_{y}\left(\left(X_{m}^{\prime}\right)_{\kappa(y)}\right), Y_{y}\right)=0 .
$$

In order to solve this problem, the use of an arbitrary connection in the fibration $\kappa: F \rightarrow$ $M$ is required. Thus, let $\nabla$ be a connection in $\kappa: F \rightarrow M$, and $\mathcal{Y}_{\eta}^{\nabla}$ the corresponding locally decomposable $m$-vector field on $F$ such that $i\left(\mathcal{Y}_{\eta}^{\nabla}\right) \omega=1$. As is well-known (see Appendix and Section 2.2), the connection $\nabla$ induces a splitting

$$
\Lambda^{k} \mathrm{~T}^{*} F=\bigoplus_{p, q=0, \ldots, k ; p+q=k}\left(\Lambda^{p} \mathrm{H}^{*}(\nabla) \oplus \Lambda^{q} \mathrm{~V}^{*}(\kappa)\right)
$$

where $\mathrm{H}(\nabla) \rightarrow F$ is the horizontal subbundle associated with the connection $\nabla$ and $\mathrm{V}(\kappa) \rightarrow F$ is the vertical subbundle of the fibration $\kappa: F \rightarrow M$. Thus, we have that

$$
\Omega=\Omega^{(m, 1)}+\Omega^{\nabla},
$$

$\Omega^{(m, 1)}$ being a $(m+1)$-form of bidegree $(m, 1)$ and $\Omega^{\nabla}$ a $(m+1)$-form. Moreover, as a straightforward consequence of Proposition 2, we have that: 
Proposition $5 \Omega^{(m, 1)}=\omega \wedge \gamma_{\eta}^{\nabla}$, where $\gamma_{\eta}^{\nabla}:=i\left(\mathcal{Y}_{\eta}^{\nabla}\right) \Omega$. Hence $\Omega=\Omega^{\nabla}+\omega \wedge \gamma_{\eta}^{\nabla}$.

In what follows, we assume that the following condition holds:

Assumption 2 The $(m+1)$-form $\Omega^{\nabla}$ is of bidegree $(m-1,2)$.

By Proposition 5, this is equivalent to demanding that

$$
i\left(Z_{1}\right) i\left(Z_{2}\right) i\left(Z_{3}\right) \Omega=0 \text {, for every } Z_{1}, Z_{2}, Z_{3} \in \mathfrak{X}^{\mathrm{V}(\kappa)}(F) .
$$

Remark 1 The above assumption is justified because this is the situation in the Lagrangian and Hamiltonian formalism of field theories (see Propositions 7 and 11).

\subsection{Conditions for the existence of solutions on a submanifold of the total space}

Taking into account the above considerations, the necessary and sufficient condition for the existence of solutions to the problem posed in the Statement 10 arises from the results obtained in Sections 2.2 and 2.3. The key consists in working at every point of the manifolds involved in this problem. Thus, if $y \in C$, the following identifications can be made:

$$
\mathcal{E} \equiv \mathrm{T}_{\kappa(y)} M \quad, \quad \mathcal{W} \equiv \mathrm{T}_{y} F \quad, \quad \mathcal{C} \equiv \mathrm{T}_{y} C \quad, \quad \mathrm{~V}(\sigma) \equiv \mathrm{V}_{y}(\kappa)
$$

Then we may consider the $\mathbb{R}$-linear map

$$
b_{\Omega}^{\nabla}(y): \mathrm{T}_{\kappa(y)}^{*} M \otimes \mathrm{T}_{y} C \rightarrow\left(\mathrm{T}_{\kappa(y)}^{*} M \otimes H_{y}(\nabla)\right) \times V_{y}^{*}(\kappa)
$$

defined by

$$
\left.b_{\Omega}^{\nabla}(y)\left(\mathbf{h}_{y}\right)=\left.\left(\left(\mathbf{h}_{y}\right)_{\nabla}^{H}, i\left(i\left(\widetilde{\left(\mathbf{h}_{y}\right)_{\nabla}^{V}}\right]^{t}\right)\left(\mathcal{Y}_{\eta}^{\nabla}(y)\right)\right)(\Omega(y))\right|_{\mathrm{V}_{y}(\kappa)}\right) .
$$

Therefore, Theorem 1 and Corollary 1 lead to the following results:

Theorem 3 Let $y \in C$. Then, there exists a linear map $\mathbf{h}_{y} \in \mathrm{T}_{\kappa(y)}^{*} M \otimes \mathrm{T}_{y} C$ such that (11) and (12) hold if, and only if,

$$
b_{\Omega}^{\nabla}(y)\left(\mathbf{h}_{y}\right)=\left(\left(\mathrm{T}_{y} \kappa_{\mathrm{H}(\nabla)}\right)^{-1},-\left.\gamma_{\eta}^{\nabla}(y)\right|_{\mathrm{V}_{y}(\kappa)}\right)
$$

where $\left(\mathrm{T}_{y} \kappa_{\mathrm{H}(\nabla)}\right)^{-1}: \mathrm{T}_{\kappa(y)} M \rightarrow \mathrm{H}_{y}(\nabla)$ is the horizontal lift at $y$ associated with the connection $\nabla$. (Observe that $\left.\left(\mathrm{T}_{y} \kappa_{\mathrm{H}(\nabla)}\right)^{-1} \in \mathrm{T}_{\kappa(y)}^{*} M \otimes \mathrm{H}_{y}(\nabla)\right)$.

Corollary 2 If $y \in C$, and $\mathbf{h}_{y} \in \mathrm{T}_{\kappa(y)}^{*} M \otimes \mathrm{T}_{y} C$, then (11) and (12) hold if, and only if,

$$
\left.\left(\mathbf{h}_{y}\right)_{\nabla}^{H}=\left(\mathrm{T}_{y} \kappa_{\mathrm{H}(\nabla)}\right)^{-1}, \quad\left[i\left(i\left(\widetilde{\left[\left(\mathbf{h}_{y}\right)_{\nabla}^{V}\right.}\right]^{t}\right)\left(\mathcal{Y}_{\eta}^{\nabla}(y)\right)\right)(\Omega(y))\right]\left.\right|_{\mathrm{V}_{y}(\kappa)}=-\left.\left(\gamma_{\eta}^{\nabla}(y)\right)\right|_{\mathrm{V}_{y}(\kappa)} .
$$

Remark 2 If $y \in C$, let $\mathrm{V}_{y}(\kappa)^{0} \subseteq \mathrm{T}_{y}^{*} \mathcal{W}$ be the annihilator of the vertical subspace $\mathrm{V}_{y}(\kappa)$ at the point $y$. Then we have that

$$
\left[\operatorname{Lin}\left(\mathrm{T}_{\kappa(y)} M, \mathrm{H}_{y}(\nabla)\right)\right]^{*} \cong \mathrm{T}_{\kappa(y)} M \otimes \mathrm{H}_{y}^{*}(\nabla) \cong \mathrm{T}_{\kappa(y)} M \otimes \mathrm{V}_{y}(\kappa)^{0} \cong \operatorname{Lin}\left(\mathrm{T}_{\kappa(y)}^{*} M, \mathrm{~V}_{y}(\kappa)^{0}\right)
$$


If $\mathbf{h}_{y}: \mathrm{T}_{\kappa(y)} M \rightarrow \mathrm{H}_{y}(\nabla)$ and $\mathbf{h}_{y}^{*}: \mathrm{T}_{\kappa(y)}^{*} M \rightarrow \mathrm{V}_{y}(\kappa)^{0}$ are linear maps, $\left\{\left(X_{1}\right)_{\kappa(y)}, \ldots,\left(X_{m}\right)_{\kappa(y)}\right\}$ is a basis of $\mathrm{T}_{\kappa(y)} M$ such that $\left\{\alpha_{\kappa(y)}^{1}, \ldots, \alpha_{\kappa(y)}^{m}\right\}$ is the dual basis of $\mathrm{T}_{\kappa(y)}^{*} M$, and

$$
\eta(\kappa(y))=\left(\alpha^{1}\right)_{\kappa(y)} \wedge \cdots \wedge\left(\alpha^{m}\right)_{\kappa(y)}, \quad \mathcal{X}_{\eta}(\kappa(y))=\left(X_{1}\right)_{\kappa(y)} \wedge \cdots \wedge\left(X_{m}\right)_{\kappa(y)},
$$

then, taking $\left(Y_{i}\right)_{y}=\left(\mathrm{T}_{y} \kappa_{\mathrm{H}(\nabla)}\right)\left(\left(X_{i}\right)_{\kappa(y)}\right)$ and $\beta_{y}^{i}=\alpha_{\kappa(y)}^{i} \circ \mathrm{T}_{y} \kappa$, for all $i \in\{1, \ldots, m\}$, we deduce that $\left\{\left(Y_{1}\right)_{y}, \ldots,\left(Y_{m}\right)_{y}\right\}$ and $\left\{\beta_{y}^{1}, \ldots, \beta_{y}^{m}\right\}$ are a basis of $\mathrm{H}_{y}(\nabla)$ and $\mathrm{V}_{y}(\kappa)^{0}$, respectively. Moreover, if

$$
\mathbf{h}_{y}\left(\left(X_{i}\right)_{\kappa(y)}\right)=\left(\mathbf{h}_{y}\right)_{i}^{j}\left(Y_{j}\right)_{y}, \quad \mathbf{h}_{y}^{*}\left(\alpha_{\kappa(y)}^{i}\right)=\left(\mathbf{h}_{y}^{*}\right)_{j}^{i} \beta_{y}^{j}, \quad \text { for every } i \in\{1, \ldots, m\}
$$

it follows that $<\mathbf{h}_{y}^{*}, \mathbf{h}_{y}>=\mathbf{h}_{y}^{*}\left(\mathbf{h}_{y}\right)=\left(\mathbf{h}_{y}\right)_{i}^{j}\left(\mathbf{h}_{y}^{*}\right)_{j}^{i}$.

Now, if $y \in C$, the orthogonal complement $\left(\mathrm{T}_{y}^{\perp} C\right)_{\Omega}^{\nabla}$ with respect to $\Omega$ and $\nabla$ is the subspace of $\left(\mathrm{T}_{\kappa(y)} M \otimes \mathrm{V}_{y}(\kappa)^{0}\right) \times \mathrm{V}_{y}(\kappa)$ defined by

$$
\left(\mathrm{T}_{y}^{\perp} C\right)_{\Omega}^{\nabla}=\left(\operatorname{Im} b_{\Omega}^{\nabla}(y)\right)^{0} .
$$

As in Theorem 2, from Theorem 3 we obtain

Theorem 4 Let $y \in C$. Then, there exists a linear map $\mathbf{h}_{y} \in \mathrm{T}_{\kappa(y)}^{*} M \otimes \mathrm{T}_{y} C$ such that

$$
\left.\left(\mathbf{h}_{y}\right)_{\nabla}^{H}=\left(\mathrm{T}_{y} \kappa_{\mathrm{H}(\nabla)}\right)^{-1}, \quad\left[i\left(i\left(\widetilde{\left(\mathbf{h}_{y}\right)_{\nabla}^{V}}\right]^{t}\right)\left(\mathcal{Y}_{\eta}^{\nabla}(y)\right)\right)(\Omega(y))\right]\left.\right|_{\mathrm{V}_{y}(\kappa)}=-\left.\left(\gamma_{\eta}^{\nabla}(y)\right)\right|_{\mathrm{V}_{y}(\kappa)}
$$

if, and only if,

$$
\mathbf{h}_{y}^{*}\left(\mathrm{~T}_{y} \kappa_{\mathrm{H}(\nabla)}\right)^{-1}-\gamma_{\eta}^{\nabla}(y)\left(Z_{y}\right)=0, \quad \text { for every }\left(\mathbf{h}_{y}^{*}, Z_{y}\right) \in\left(\mathrm{T}_{y}^{\perp} C\right)_{\Omega}^{\nabla} .
$$

Note that if $\left(\mathrm{T}_{y}^{\perp} C\right)_{\Omega}^{\nabla}=\{0\}$ then it is clear that (15) holds. Thus, from Proposition 3 we have:

Proposition 6 If the $(m+1)$-form $\Omega^{\nabla}$ on $F$ given by $\Omega^{\nabla}=\Omega-\omega \wedge \gamma_{\eta}^{\nabla}$ is 1-nondegenerate, that is, the map $b_{\Omega^{\nabla}}: \mathrm{T} F \rightarrow \Lambda^{m} \mathrm{~T}^{*} F$ is injective, then

$$
\left(\mathrm{T}_{y}^{\perp} F\right)_{\Omega}^{\nabla}=\{0\}, \quad \text { for every } y \in F .
$$

\subsection{The pre-multisymplectic constraint algorithm}

Now we apply the above results in order to solve the problem stated in Section 3.1. The procedure is algorithmic, and gives a sequence of subsets $\left\{C_{i}\right\}$ of $F$. Then, we assume that:

Assumption 3 Every subset $C_{i}$ of this sequence is a regular submanifold of $F$, and its natural injection is an embedding.

Thus, we consider the submanifold $C_{1} \hookrightarrow F$ where a solution exists, that is,

$$
\begin{array}{r}
C_{1}=\left\{y \in F \mid \exists \mathbf{h}_{y} \in \underset{\operatorname{Lin}\left(\mathrm{T}_{\kappa}(y)\right.}{\operatorname{Li}} M, \mathrm{~T}_{y} F\right) \text { such that }\left(\mathbf{h}_{y}\right)_{\nabla}^{H}=\left(\mathrm{T}_{y} \kappa_{\mathrm{H}(\nabla)}\right)^{-1}, \\
\left.\left.\left[i\left(i\left(\left[\left(\mathbf{h}_{y}\right)_{\nabla}^{V}\right]^{*}\right)\left(\mathcal{Y}_{\eta}^{\nabla}(y)\right)\right)(\Omega(y))\right]\right|_{\mathrm{V}_{y}(\kappa)}=-\left.\left(\gamma_{\eta}^{\nabla}(y)\right)\right|_{\mathrm{V}_{y}(\kappa)}\right\} .
\end{array}
$$


Then, using the results of Section 3.2 we deduce that there is a locally decomposable section $\mathcal{X}_{1}$ of the vector bundle $\Lambda^{m} \mathrm{~T}_{C_{1}} F \rightarrow C_{1}$ such that $\left.\left(i\left(\mathcal{X}_{1}\right) \omega\right)\right|_{C_{1}}=1$ and $\left.\left(i\left(\mathcal{X}_{1}\right) \Omega\right)\right|_{C_{1}}=0$. However, in general, $\mathbf{h}_{y}\left(\mathrm{~T}_{\kappa(y)} M\right)$ is not a subspace of $\mathrm{T}_{y} C_{1}$ and then $\mathcal{X}_{1}$ is not tangent to $C_{1}$ or, in other words, in general, $\mathcal{X}_{1}$ is not a connection in the fibration $\kappa: F \rightarrow M$ along $C_{1}$. Therefore, we consider the submanifold

$$
\begin{aligned}
& C_{2}=\left\{y_{1} \in C_{1} \mid \exists \mathbf{h}_{y_{1}} \in \operatorname{Lin}\left(\mathrm{T}_{\kappa\left(y_{1}\right)} M, \mathrm{~T}_{y_{1}} C_{1}\right) \text { such that }\left(\mathbf{h}_{y_{1}}\right)_{\nabla}^{H}=\left(\mathrm{T}_{y_{1}} \kappa_{\mathrm{H}(\nabla)}\right)^{-1},\right. \\
& {\left[\left.i\left(i\left(\left[\left(\widehat{\left.\mathbf{h}_{y_{1}}\right)_{\nabla}^{V}}\right]^{*}\right)\left(\mathcal{Y}_{\eta}^{\nabla}\left(y_{1}\right)\right)\right)\left(\Omega\left(y_{1}\right)\right)\right]\right|_{\mathrm{V}_{y_{1}}(\kappa)}=-\left.\left(\gamma_{\eta}^{\nabla}\left(y_{1}\right)\right)\right|_{\mathrm{V}_{y_{1}}(\kappa)}\right\} \text {. }}
\end{aligned}
$$

Then, there is a locally decomposable section $\mathcal{X}_{2}$ of the vector bundle $\Lambda^{m} \mathrm{~T}_{C_{2}} C_{1} \rightarrow C_{2}$ such that $\left.\left(i\left(\mathcal{X}_{2}\right) \omega\right)\right|_{C_{2}}=1$ and $\left.\left(i\left(\mathcal{X}_{2}\right) \Omega\right)\right|_{C_{2}}=0$. However, in general, $\mathcal{X}_{2}$ is not a connection in the fibration $\kappa: F \rightarrow M$ along $C_{2}$. Following this process, we obtain a sequence of constraint submanifolds

$$
\cdots \stackrel{j_{i+1}^{i}}{\longrightarrow} C_{i} \stackrel{j_{i}^{i-1}}{\hookrightarrow} \cdots \stackrel{j_{2}^{1}}{\longrightarrow} C_{1} \stackrel{j_{1}}{\hookrightarrow} C_{0} \equiv F .
$$

For every $i \geq 1, C_{i}$ is called the $i$ th constraint submanifold.

This procedure is called the pe-multisymplectic constraint algorithm. We have two possibilities:

- There exists an integer $k>0$ such that $\operatorname{dim} C_{k} \leq m-1$. This means that the equations have no solution on a submanifold of $F$.

- There exists an integer $k>0$ such that $C_{k+1}=C_{k} \equiv C_{f}$. In such a case, there exists a connection $\mathcal{X}_{f}$ in the fibration $\kappa: F \rightarrow M$ along $C_{f}$ such that

$$
i\left(\mathcal{X}_{f}\left(y_{f}\right)\right)\left(\Omega\left(y_{f}\right)\right)=0, \quad \text { for every } y_{f} \in C_{f} .
$$

In this case, $C_{f}$ is called the final constraint submanifold. This is the situation which is interesting to us. Note that the existence of a connection in the fibration $\kappa: F \rightarrow M$ along $C_{f}$ implies that $\kappa\left(C_{f}\right)$ is an open subset of $M$ and that $\left.\kappa\right|_{C_{f}}: C_{f} \rightarrow \kappa\left(C_{f}\right)$ is a fibration (see Remark 5 in the Appendix). In particular, $\operatorname{dim} C_{f} \geq m$.

Next we give an intrinsic characterization of the constraints which define the constraint submanifolds $C_{i}$. For this purpose, we consider the vector bundle over $F$,

$$
W(\kappa, \nabla)=\left(\kappa^{*}\left(\mathrm{~T}^{*} M\right) \otimes \mathrm{H}(\nabla)\right) \oplus_{F} \mathrm{~V}^{*}(\kappa)
$$

whose fiber over the point $y \in F$ is

$$
W_{y}(\kappa, \nabla)=\left(\mathrm{T}_{\kappa(y)}^{*} M \otimes \mathrm{H}_{y}(\nabla)\right) \times \mathrm{V}_{y}^{*}(\kappa) \cong \operatorname{Lin}\left(\mathrm{T}_{\kappa(y)} M, \mathrm{H}_{y}(\nabla)\right) \times \mathrm{V}_{y}^{*}(\kappa) .
$$

The horizontal lift associated with the connection $\nabla$ and the 1 -form $\gamma_{\eta}^{\nabla}$ induce a section $\left(\left(\mathrm{T} \kappa_{\mathrm{H}(\nabla)}\right)^{-1}\right.$, $\left.-\left.\left(\gamma_{\eta}^{\nabla}\right)\right|_{\mathrm{V}(\kappa)}\right)$ of this vector bundle given by

$$
\left(\left(\mathrm{T} \kappa_{\mathrm{H}(\nabla)}\right)^{-1},-\left.\left(\gamma_{\eta}^{\nabla}\right)\right|_{\mathrm{V}(\kappa)}\right)(y)=\left(\left(\mathrm{T}_{y} \kappa_{\mathrm{H}(\nabla)}\right)^{-1},-\left.\left(\gamma_{\eta}^{\nabla}(y)\right)\right|_{\mathrm{V}_{y}(\kappa)}\right), \quad \text { for every } y \in F .
$$

Furthermore, let $W_{C_{i}}(\kappa, \nabla)$ be the vector bundle over the submanifold $C_{i}$ whose fiber at the point $y_{i} \in C_{i}$ is $W_{y_{i}}(\kappa, \nabla)$. Moreover, we may consider the orthogonal complement $\left(\mathrm{T}_{y_{i}}^{\perp} C_{i}\right)_{\Omega}^{\nabla}$ of $\mathrm{T}_{y_{i}} C_{i}$ with respect to $\Omega$ and $\nabla$ given by (see (14))

$$
\begin{array}{r}
\left(\mathrm{T}_{y_{i}}^{\perp} C_{i}\right)_{\Omega}^{\nabla}=\left\{\left(\mathbf{h}_{y_{i}}^{*}, Z_{y_{i}}\right) \in \operatorname{Lin}\left(\mathrm{T}_{\kappa\left(y_{i}\right)}^{*} M, \mathrm{~V}_{y_{i}}(\kappa)^{0}\right) \times \mathrm{V}_{y_{i}}(\kappa) \mid \mathbf{h}_{y_{i}}^{*}\left(\left(\mathbf{h}_{y_{i}}\right)_{\nabla}^{H}\right)+\right. \\
\left.i\left(Z_{y_{i}}\right) i\left(i\left(\left[\left(\mathbf{h}_{y_{i}}\right)_{\nabla}^{V}\right]^{t}\right)\left(\mathcal{Y}_{\eta}^{\nabla}(y)\right)\right)\left(\Omega\left(y_{i}\right)\right)=0, \text { for every } \mathbf{h}_{y_{i}} \in \operatorname{Lin}\left(\mathrm{T}_{\kappa\left(y_{i}\right)} M, \mathrm{~T}_{y_{i}} C_{i}\right)\right\} .
\end{array}
$$


Note that $\left(\mathrm{T}_{y_{i}}^{\perp} C_{i}\right)_{\Omega}^{\nabla} \subseteq W_{y_{i}}^{*}(\kappa, \nabla)$. Furthermore, if $\left(\mathrm{T}^{\perp} C_{i}\right)_{\Omega}^{\nabla}$ is a vector subbundle of rank $r$ of $W_{C_{i}}^{*}(\kappa, \nabla)$ (that is, the dimension of $\left(\mathrm{T}_{y_{i}}^{\perp} C_{i}\right)_{\Omega}^{\nabla}$ is $r$, for every $y_{i} \in C_{i}$ ) then one may choose a set of $r$ local sections $\left\{\left(\mathbf{h}_{1}^{*}, Z_{1}\right), \ldots,\left(\mathbf{h}_{r}^{*}, Z_{r}\right)\right\}$ of the vector bundle $W^{*}(\kappa, \nabla) \rightarrow F$ such that $\left\{\left.\left(\mathbf{h}_{1}^{*}, Z_{1}\right)\right|_{C_{i}}, \ldots,\left.\left(\mathbf{h}_{r}^{*}, Z_{r}\right)\right|_{C_{i}}\right\}$ is a local basis of the space $\Gamma\left(\left(\mathrm{T}^{\perp} C_{i}\right)_{\Omega}^{\nabla}\right)$ of sections of the vector subbundle $\left(\mathrm{T}^{\perp} C_{i}\right)_{\Omega}^{\nabla} \rightarrow C_{i}$. In addition, using Theorem 4 , we deduce

Theorem 5 Every submanifold $C_{i}(i \geq 1)$ in the sequence (16) may be defined as

$$
C_{i}=\left\{y_{i-1} \in C_{i-1} \mid\left\langle\left(\left(\mathrm{T} \kappa_{\mathrm{H}(\nabla)}\right)^{-1},-\left.\left(\gamma_{\eta}^{\nabla}\right)\right|_{\mathrm{V}(\kappa)}\right)\left(y_{i-1}\right),\left(\mathrm{T}_{y_{i-1}}^{\perp} C_{i-1}\right)_{\Omega}^{\nabla}\right\rangle=0\right\}
$$

Therefore, if $\left(\mathrm{T}^{\perp} C_{i-1}\right)_{\Omega}^{\nabla}$ is a vector subbundle of rank $r$ of $W_{C_{i-1}}^{*}(\kappa, \nabla)$ and $\left\{\left(\mathbf{h}_{1}^{*}, Z_{1}\right)^{(i-1)}, \ldots\right.$, $\left.\left(\mathbf{h}_{r}^{*}, Z_{r}\right)^{(i-1)}\right\}$ is a set of sections of the vector bundle $W^{*}(\kappa, \nabla) \rightarrow F$ spanning locally the space $\Gamma\left(\left(\mathrm{T}^{\perp} C_{i-1}\right)_{\Omega}^{\nabla}\right)$, then $C_{i}$, is defined locally, as a submanifold of $C_{i-1}$, as the zero set of the functions $\xi_{j}^{(i)} \in C^{\infty}(F)$ given by

$$
\xi_{j}^{(i)}=\left(\left(\mathrm{T} \kappa_{\mathrm{H}(\nabla)}\right)^{-1},-\left.\left(\gamma_{\eta}^{\nabla}\right)\right|_{\mathrm{V}(\kappa)}\right)\left(\left(\mathbf{h}_{j}^{*}, Z_{j}\right)^{(i-1)}\right) .
$$

These functions are called $i$ th-generation constraints.

\subsection{The integrability algorithm}

Suppose that after applying the premultisymplectic constraint algorithm we have a final constraint submanifold $C_{f} \hookrightarrow F$ and a connection defined by the multivector field $\mathcal{X}_{f}$ in the fibration $\kappa: F \rightarrow$ $M$ along $C_{f}$ such that (9) holds on $C_{f}$, that is,

$$
i\left(\mathcal{X}_{f}(y)\right) \Omega(y)=0, \quad \text { for every } y \in C_{f} .
$$

However, $\mathcal{X}_{f}$ is not, in general, a flat connection. Nevertheless, in many cases, one may find a submanifold $\mathcal{I}_{f}$ of $C_{f}$ such that $\left.\left(\mathcal{X}_{f}\right)\right|_{\mathcal{I}_{f}}$ is a flat connection in the fibration $\kappa: F \rightarrow M$ along $\mathcal{I}_{f}$ and (9) holds for $\left.\left(\mathcal{X}_{f}\right)\right|_{\mathcal{I}_{f}}$.

Next we present an algorithm which enables us to find this submanifold (which is an adapted version of that given in [10]). This is a local algorithm, that is, we are in fact working on suitable open sets in $C_{f}$. Hence, let $\mathcal{X}_{f} \equiv \bigwedge_{\mu=1}^{m} X_{\mu}$ be a solution to (17).

- Integrability condition: The condition that $\mathcal{X}_{f}$ is flat is equivalent to demanding that the distribution spanned by $X_{1}, \ldots, X_{m}$ is involutive. Then, if $c_{f}=\operatorname{dim} C_{f}$, let $Z_{1}, \ldots, Z_{n-m} \in$ $\mathfrak{X}(F)$, such that $\left\{X_{1}, \ldots, X_{m}, Z_{1}, \ldots, Z_{c_{f}-m}\right\}$ is a local basis of the module of vector fields on $C_{f}$. Therefore, for every pair $X_{\mu}, X_{\nu}(1 \leq \mu, \nu \leq m)$ we have

$$
\left[X_{\mu}, X_{\nu}\right]=f_{\mu \nu}^{\rho} X_{\rho}+\zeta_{\mu \nu}^{l} Z_{l}
$$

for some functions $f_{\mu \nu}^{\rho}, \zeta_{\mu \nu}^{l}$. Consider the system $\zeta_{\mu \nu}^{l}=0$ and let

$$
\mathcal{I}_{1}=\left\{y \in C_{f} ; \zeta_{\mu \nu}^{l}(y)=0, \forall \mu, \nu, l\right\} .
$$

We have three options:

1. $\mathcal{I}_{1}=C_{f}$. Then the distribution spanned by $X_{1}, \ldots, X_{m}$ is involutive, and $\left.\left(\mathcal{X}_{f}\right)\right|_{C_{f}}$ is a flat connection in the fibration $\kappa: F \rightarrow M$ along $C_{f}$. 
2. $\mathcal{I}_{1}=\emptyset$. Then the distribution spanned by $X_{1}, \ldots, X_{m}$ is not involutive at any point in $C_{f}$, and hence the $m$-vector field $\mathcal{X}_{f}$ is not integrable.

3. $\mathcal{I}_{1}$ is a proper subset of $C_{f}$. In this case we assume that $\mathcal{I}_{1}$ is a closed submanifold of $C_{f}$ and the functions $\zeta_{\mu \nu}^{l}$ are the constraints locally defining $\mathcal{I}_{1}$. The distribution spanned by $X_{1}, \ldots, X_{m}$ is involutive on $\mathcal{I}_{1}$; that is, the $m$-vector field $\mathcal{X}_{f}$ is integrable on $\mathcal{I}_{1}$.

If $\mathcal{X}_{f}$ is tangent to $\mathcal{I}_{1}$, then $\left.\left(\mathcal{X}_{f}\right)\right|_{\mathcal{I}_{1}}$ defines a flat connection in $\kappa: F \rightarrow M$ along $\mathcal{I}_{1}$ and (9) holds on $\mathcal{I}_{1}$ which implies that the problem is solved. Nevertheless, this is not the case in general, so we need the following:

- Tangency condition: Consider the set

$$
\mathcal{I}_{2}:=\left\{y \in \mathcal{I}_{1} ; \mathcal{X}_{f}(y) \in \Lambda^{m} \mathrm{~T}_{y} \mathcal{I}_{1}\right\}
$$

For $\mathcal{I}_{2}$ we have the same problem, so we define inductively, for $i>1$,

$$
\mathcal{I}_{i}:=\left\{y \in \mathcal{I}_{i-1} ; \mathcal{X}_{f}(y) \in \Lambda^{m} \mathrm{~T}_{y} \mathcal{I}_{i-1}\right\}
$$

and assume that we obtain a sequence $\ldots \subset \mathcal{I}_{i} \subset \ldots \subset \mathcal{I}_{1} \subset C_{f}$ such that $\mathcal{I}_{i}$ is a non-empty (closed) submanifold of $F$, for all $i$, or $\mathcal{I}_{i}=\emptyset$, for some $i$.

Observe that the locally decomposable $m$-vector field $\mathcal{X}_{f}=X_{1} \wedge \ldots \wedge X_{m}$ is tangent to $\mathcal{I}_{i}$ (with $\mathcal{I}_{i} \neq \emptyset$ ) if, and only if, $X_{\mu}$ is tangent to $\mathcal{I}_{i}$, for every $\mu$.

Thus, using the constraints, we have that, if $\left\{\zeta_{\alpha_{i}}^{(i)}\right\}$ is a basis of constraints defining locally $\mathcal{I}_{i}$ in $\mathcal{I}_{i-1}$, the tangency condition is $0 \underset{\mathcal{I}_{i}}{=} X_{\mu}\left(\zeta_{\alpha_{i}}^{(i)}\right)$ (for every $\mu, \alpha_{i}$ ), that is, we have

$$
\mathcal{I}_{i+1}:=\left\{y \in \mathcal{I}_{i} ; X_{\mu}\left(\zeta_{\alpha_{i}}^{(i)}\right)(y)=0, \forall \mu, \alpha_{i}\right\}, \quad \text { for every } i \geq 1
$$

The above algorithm ends at step $f$ in one of the following two options:

1. $\operatorname{dim} \mathcal{I}_{f} \leq m-1$. In such a case, we deduce that it is not possible to find a submanifold $\mathcal{I}$ of $C_{f}$ such that $\left.\left(\mathcal{X}_{f}\right)\right|_{\mathcal{I}}$ is a flat connection in the fibration $\kappa: F \rightarrow M$ along $\mathcal{I}$. Therefore, we must consider (if it exists) another connection $\mathcal{X}_{f}^{\prime}$ along $C_{f}$ such that $i\left(\mathcal{X}_{f}^{\prime}(y)\right) \Omega(y)=0$, for every $y \in C_{f}$, and then we must repeat the above procedure.

2. $\mathcal{I}_{f+1}=\mathcal{I}_{f}$. In this case $\mathcal{I}_{f}$ is a submanifold of $F$ and we deduce that $\left.\left(\mathcal{X}_{f}\right)\right|_{\mathcal{I}_{f}}$ is a flat connection in the fibration $\kappa: F \rightarrow M$ along $\mathcal{I}_{f}$ such that $i\left(\mathcal{X}_{f}(y)\right)(\Omega(y))=0$, for every $y \in \mathcal{I}_{f}$. Thus, the problem is solved. As in Section 3.3 we remark that the existence of a connection in the fibration $\kappa: F \rightarrow M$ along $\mathcal{I}_{f}$ implies that $\kappa\left(\mathcal{I}_{f}\right)$ is an open subset of $M$ and that $\left.\kappa\right|_{\mathcal{I}_{f}}: \mathcal{I}_{f} \rightarrow \kappa\left(\mathcal{I}_{f}\right)$ is a fibration. In particular, $\operatorname{dim} \mathcal{I}_{f} \geq m$.

We will call this procedure the integrability algorithm for decomposable $m$-vector fields.

\section{Application to Lagrangian and Hamiltonian field theories}

\subsection{Lagrangian and Hamiltonian field theories}

(For details on the construction of the Lagrangian and Hamiltonian formalisms of field theories, see for instance, [2, 3], 9], [10, 11], 12], 13], 21], 25], 26], 39], 41].) 
A first-order classical field theory is described by its configuration fibre bundle $\pi: E \rightarrow M$ and a Lagrangian density which is a $\bar{\pi}^{1}$-semibasic $m$-form, $\mathcal{L}$, on $J^{1} \pi$ (the first-order jet bundle of $\pi: E \rightarrow M) . \mathcal{L}$ is usually written as $\mathcal{L}=L\left(\bar{\pi}^{1 *} \eta\right) \equiv L \omega$, where $L \in \mathrm{C}^{\infty}\left(J^{1} \pi\right)$ is the Lagrangian function associated with $\mathcal{L}$ and $\omega$, and $\pi^{1}: J^{1} \pi \rightarrow E$ and $\bar{\pi}^{1}:=\pi \circ \bar{\pi}: J^{1} \pi \rightarrow M$ are the natural projections. The Poincaré-Cartan $m$ and $(m+1)$-forms associated with the Lagrangian density $\mathcal{L}$ are defined using the vertical endomorphism $\mathcal{V}$ of the bundle $J^{1} \pi$

$$
\Theta_{\mathcal{L}}:=i(\mathcal{V}) \mathcal{L}+\mathcal{L} \in \Omega^{m}\left(J^{1} \pi\right) \quad ; \quad \Omega_{\mathcal{L}}:=-\mathrm{d} \Theta_{\mathcal{L}} \in \Omega^{m+1}\left(J^{1} \pi\right)
$$

Then a Lagrangian system is a couple $\left(J^{1} \pi, \Omega_{\mathcal{L}}\right)$. The Lagrangian system is regular if $\Omega_{\mathcal{L}}$ is 1 nondegenerate. Elsewhere it is called singular. In a natural chart of coordinates $\left(x^{\alpha}, y^{A}, v_{\alpha}^{A}\right)$ in $J^{1} \pi$ (adapted to the bundle structure, and such that $\omega=\mathrm{d} x^{1} \wedge \ldots \wedge \mathrm{d} x^{m} \equiv \mathrm{d} x^{m}$ ) we have

$$
\begin{aligned}
\Omega_{\mathcal{L}}= & -\frac{\partial^{2} L}{\partial v_{\nu}^{B} \partial v_{\alpha}^{A}} \mathrm{~d} v_{\nu}^{B} \wedge \mathrm{d} y^{A} \wedge \mathrm{d}^{m-1} x_{\alpha}-\frac{\partial^{2} L}{\partial y^{B} \partial v_{\alpha}^{A}} \mathrm{~d} y^{B} \wedge \mathrm{d} y^{A} \wedge \mathrm{d}^{m-1} x_{\alpha} \\
& +\frac{\partial^{2} L}{\partial v_{\nu}^{B} \partial v_{\alpha}^{A}} v_{\alpha}^{A} \mathrm{~d} v_{\nu}^{B} \wedge \mathrm{d}^{m} x+\left(\frac{\partial^{2} L}{\partial y^{B} \partial v_{\alpha}^{A}} v_{\alpha}^{A}-\frac{\partial L}{\partial y^{B}}+\frac{\partial^{2} L}{\partial x^{\alpha} \partial v_{\alpha}^{B}}\right) \mathrm{d} y^{B} \wedge \mathrm{d}^{m} x
\end{aligned}
$$

(where $\mathrm{d}^{m} x=\mathrm{d} x^{1} \wedge \ldots \wedge \mathrm{d} x^{m}$ and $\left.\mathrm{d}^{m-1} x^{\alpha} \equiv i\left(\frac{\partial}{\partial x^{\alpha}}\right) \mathrm{d}^{m} x\right)$. Locally, the regularity condition is equivalent to $\operatorname{det}\left(\frac{\partial^{2} L}{\partial v_{\alpha}^{A} \partial v_{\nu}^{B}}(\bar{y})\right) \neq 0$, for every $\bar{y} \in J^{1} \pi$.

The Lagrangian problem associated with a Lagrangian system $\left(J^{1} \pi, \Omega_{\mathcal{L}}\right)$ consists in finding sections $\phi \in \Gamma(M, E)$ (where $\Gamma(M, E)$ denotes the set of sections of $\pi$ ), such that

$$
\left(j^{1} \phi\right)^{*} i(X) \Omega_{\mathcal{L}}=0 \quad, \quad \text { for every } X \in \mathfrak{X}\left(J^{1} \pi\right)
$$

In natural coordinates this is equivalent to demanding that $\phi$ satisfies the Euler-Lagrange equations. The problem of finding these sections can be formulated equivalently as follows: to find the integral sections of a class of holonomic $m$-vector fields $\left\{\mathcal{X}_{\mathcal{L}}\right\} \subset \mathfrak{X}^{m}\left(J^{1} \pi\right)$, such that

$$
i\left(\mathcal{X}_{\mathcal{L}}\right) \Omega_{\mathcal{L}}=0 \quad, \quad \text { for every } \mathcal{X}_{\mathcal{L}} \in\left\{\mathcal{X}_{\mathcal{L}}\right\}
$$

(Holonomic means that $\mathcal{X}_{\mathcal{L}}$ is integrable and its integral sections are holonomic. This is equivalent to demanding that $\mathcal{X}_{\mathcal{L}}$ is integrable and semi-holonomic, that is, it satisfies the condition $i\left(\mathcal{X}_{\mathcal{L}}\right) \mathcal{V}=0$. Semi-holonomic (not necessarily integrable) locally decomposable $m$-vector fields which are solution to these equations are called Euler-Lagrange $m$-vector fields for $\left(J^{1} \pi, \Omega_{\mathcal{L}}\right)$.

For the Hamiltonian formalism of field theories, we take as the multimomentum bundle the manifold $J^{1} \pi^{*} \equiv \Lambda_{2}^{m} \mathrm{~T}^{*} E / \pi^{*} \Lambda^{m} \mathrm{~T}^{*} M$, where $\Lambda_{2}^{m} \mathrm{~T}^{*} E \equiv \mathcal{M} \pi$ is the bundle of $m$-forms on $E$ vanishing by the action of two $\pi$-vertical vector fields. It is a bundle $\bar{\tau}^{1}=\pi \circ \tau^{1}: J^{1} \pi^{*} \rightarrow M$, where $\tau^{1}: J^{1} \pi^{*} \rightarrow E$ is the natural projection. Natural charts of coordinates in $\mathcal{M} \pi$ and $J^{1} \pi$ (adapted to the bundle structure, and such that $\omega^{*} \equiv \bar{\tau}^{1 *} \eta=\mathrm{d} x^{1} \wedge \ldots \wedge \mathrm{d} x^{m} \equiv \mathrm{d} x^{m}$ ) are denoted by $\left(x^{\alpha}, y^{A}, p_{A}^{\alpha}, p\right)$ and $\left(x^{\alpha}, y^{A}, p_{A}^{\alpha}\right)$, respectively.

As $\mathcal{M} \pi$ is a subbundle of $\Lambda^{m} \mathrm{~T}^{*} E$ (the multicotangent bundle of $E$ of order $m$ ), then $\mathcal{M} \pi$ is endowed with canonical forms: the "tautological form" $\Theta \in \Omega^{m}(\mathcal{M} \pi)$, and the multisymplectic form $\Omega:=-\mathrm{d} \Theta \in \Omega^{m+1}(\mathcal{M} \pi)$. They are known as the multimomentum Liouville $m$ and $(m+1)$ forms. Their local expressions are

$$
\Theta=p_{A}^{\alpha} \mathrm{d} y^{A} \wedge \mathrm{d}^{m-1} x_{\alpha}+p \mathrm{~d}^{m} x \quad, \quad \Omega=-\mathrm{d} p_{A}^{\alpha} \wedge \mathrm{d} y^{A} \wedge \mathrm{d}^{m-1} x_{\alpha}-\mathrm{d} p \wedge \mathrm{d}^{m} x
$$

Now, if $\left(J^{1} \pi, \Omega_{\mathcal{L}}\right)$ is a Lagrangian system, the extended Legendre map associated with $\mathcal{L}$, $\widetilde{\mathcal{F} \mathcal{L}}: J^{1} \pi \rightarrow \mathcal{M} \pi$, is defined by: $\left.(\widetilde{\mathcal{F} L} \bar{y})\right)\left(Z_{1}, \ldots, Z_{m}\right):=\left(\Theta_{\mathcal{L}}\right)_{\bar{y}}\left(\bar{Z}_{1}, \ldots, \bar{Z}_{m}\right)$, for $\bar{y} \in J^{1} \pi$, where 
$Z_{1}, \ldots, Z_{m} \in \mathrm{T}_{\pi^{1}(\bar{y})} E$, and $\bar{Z}_{1}, \ldots, \bar{Z}_{m} \in \mathrm{T}_{\bar{y}} J^{1} \pi$ are such that $\mathrm{T}_{\bar{y}} \pi^{1} \bar{Z}_{\alpha}=Z_{\alpha}$. Then, using the natural projection $\mu: \mathcal{M} \pi \rightarrow J^{1} \pi^{*}$, we define the restricted Legendre map associated with $\mathcal{L}$ as $\mathcal{F} \mathcal{L}:=\mu \circ \overline{\mathcal{F} L}$. Their local expressions are

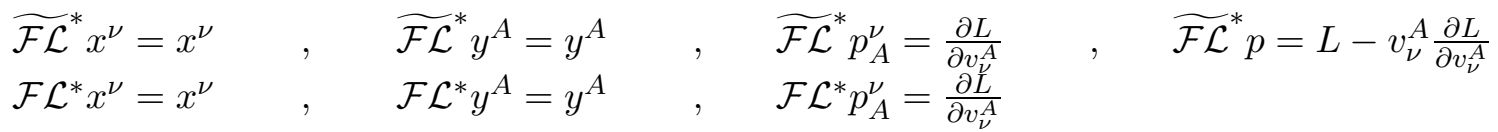

We have that $\widetilde{\mathcal{F} L}^{*} \Theta=\Theta_{\mathcal{L}}$, and $\widetilde{\mathcal{F} L}^{*} \Omega=\Omega_{\mathcal{L}}$.

$\left(J^{1} \pi, \Omega_{\mathcal{L}}\right)$ is a regular Lagrangian system if $\mathcal{F} \mathcal{L}$ is a local diffeomorphism (this definition is equivalent to that given above). Elsewhere $\left(J^{1} \pi, \Omega_{\mathcal{L}}\right)$ is a singular Lagrangian system. As a particular case, $\left(J^{1} \pi, \Omega_{\mathcal{L}}\right)$ is a hyper-regular Lagrangian system if $\mathcal{F} \mathcal{L}$ is a global diffeomorphism. A singular Lagrangian system $\left(J^{1} \pi, \Omega_{\mathcal{L}}\right)$ is almost-regular if $\mathcal{P}:=\mathcal{F} \mathcal{L}\left(J^{1} \pi\right)$ is a closed submanifold of $J^{1} \pi^{*}, \mathcal{F} \mathcal{L}$ is a submersion onto its image, and for every $\bar{y} \in J^{1} \pi$, the fibres $\mathcal{F} \mathcal{L}^{-1}(\mathcal{F} \mathcal{L}(\bar{y}))$ are connected submanifolds of $J^{1} \pi$.

If $\left(J^{1} \pi, \Omega_{\mathcal{L}}\right)$ is an almost-regular Lagrangian system then $\mathcal{P}$ is a fibre bundle over $E$ and $M$ (the natural projections are denoted by $\tau_{0}^{1}: \mathcal{P} \rightarrow E$ and $\left.\bar{\tau}_{0}^{1}:=\pi \circ \tau_{0}^{1}: \mathcal{P} \rightarrow M\right)$ and the $\mu$-transverse submanifold $\tilde{\mathcal{P}}=\widetilde{\mathcal{F} L}\left(J^{1} \pi\right) \hookrightarrow \mathcal{M} \pi$ is diffeomorphic to $\mathcal{P}$ (and we denote by $\tilde{\jmath}_{0}: \tilde{\mathcal{P}} \hookrightarrow \mathcal{M} \pi$ the natural imbedding). This diffeomorphism is denoted $\tilde{\mu}: \tilde{\mathcal{P}} \rightarrow \mathcal{P}$, and it is just the restriction of the projection $\mu$ to $\tilde{\mathcal{P}}$. Then, taking $\tilde{h}:=\tilde{\mu}^{-1}$, we define the Hamilton-Cartan $(m+1)$-form $\Omega_{h}^{0}=\left(\tilde{\jmath}_{0} \circ \tilde{h}\right)^{*} \Omega$, which verifies that $\mathcal{F} \mathcal{L}_{0}^{*} \Omega_{h}^{0}=\Omega_{\mathcal{L}}\left(\right.$ where $\mathcal{F} \mathcal{L}_{0}$ is the restriction map of $\mathcal{F} \mathcal{L}$ onto $\mathcal{P})$. Then $\tilde{h}$ is called a Hamiltonian section, and $\left(\mathcal{P}, \Omega_{\mathrm{h}}^{0}\right)$ is the Hamiltonian system associated with the almost-regular Lagrangian system $\left(J^{1} \pi, \Omega_{\mathcal{L}}\right)$ (see [26]).

If $\left(J^{1} \pi, \Omega_{\mathcal{L}}\right)$ is a hyper-regular Lagrangian system, then $\mathcal{P}=J^{1} \pi^{*}$, and the construction is the same. In addition, $\widetilde{\mathcal{F} L}\left(J^{1} \pi\right)$ is a 1-codimensional embedded submanifold of $\mathcal{M} \pi$, which is transverse to the projection $\mu$, and is diffeomorphic to $J^{1} \pi^{*}$. This diffeomorphism is $\mu^{-1}$, when $\mu$ is restricted to $\widetilde{\mathcal{F} L}\left(J^{1} \pi\right)$, and coincides with the map $h:=\widetilde{\mathcal{F} L} \circ \mathcal{F} \mathcal{L}^{-1}$, when it is restricted onto its image. $h$ is the Hamiltonian section in this case, and the associated Hamiltonian system is denoted by $\left(J^{1} \pi^{*}, \Omega_{h}\right)$, where $\Omega_{h}=h^{*} \Omega$. In a local chart of natural coordinates, the Hamiltonian section is specified by a local Hamiltonian function $H \in \mathrm{C}^{\infty}(U), U \subset J^{1} \pi^{*}$, such that $h\left(x^{\alpha}, y^{A}, p_{A}^{\alpha}\right) \equiv$ $\left(x^{\alpha}, y^{A}, p_{A}^{\alpha}, p=-H\right)$, where

$$
H\left(x^{\alpha}, y^{A}, p_{A}^{\alpha}\right)=\left(\mathcal{F} \mathcal{L}^{-1}\right)^{*}\left(v_{\alpha}^{A} \frac{\partial L}{\partial v_{\alpha}^{A}}-L\right)=p_{A}^{\alpha}\left(\mathcal{F} \mathcal{L}^{-1}\right)^{*} v_{\alpha}^{A}-\left(\mathcal{F} \mathcal{L}^{-1}\right)^{*} L
$$

and $\Omega_{h}=-\mathrm{d} p_{A}^{\alpha} \wedge \mathrm{d} y^{A} \wedge \mathrm{d}^{m-1} x_{\alpha}+\mathrm{d} H \wedge \mathrm{d}^{m} x$.

The Hamiltonian problem associated with the Hamiltonian system $\left(\mathcal{P}, \Omega_{\mathrm{h}}^{0}\right)$ (for $\left(J^{1} \pi^{*}, \Omega_{h}\right)$ is analogous), consists in finding sections $\psi_{o} \in \Gamma(M, \mathcal{P})$ such that

$$
\psi_{o}^{*} i\left(X_{0}\right) \Omega_{h}^{0}=0 \quad, \quad \text { for every } X_{0} \in \mathfrak{X}(\mathcal{P})
$$

As in the Lagrangian case, these sections are the integral sections of a class of integrable and $\bar{\tau}_{0}^{1}$-transverse $m$-vector fields $\left\{\mathcal{X}_{\mathcal{H}_{o}}\right\} \subset \mathfrak{X}^{m}(\mathcal{P})$ satisfying that

$$
i\left(\mathcal{X}_{\mathcal{H}_{o}}\right) \Omega_{h}^{0}=0 \quad, \quad \text { for every } \mathcal{X}_{\mathcal{H}_{o}} \in\left\{\mathcal{X}_{\mathcal{H}_{o}}\right\}
$$

$m$-vector fields satisfying these conditions (but not necessarily integrable) are called Hamilton-De Donder-Weyl $m$-vector fields for $\left(\mathcal{P}, \Omega_{\mathrm{h}}^{0}\right)$. 


\subsection{Lagrangian and Hamiltonian algorithms}

Let $\left(J^{1} \pi, \Omega_{\mathcal{L}}\right)$ be a Lagrangian system. If $\nabla$ is an Ehresmann connection in the fibration $\bar{\pi}^{1}: J^{1} \pi \rightarrow$ $M$, let $\mathcal{Y}_{\eta}^{\nabla}$ be the corresponding $m$-vector field on $J^{1} \pi$. Then, we have:

Proposition 7 The Poincaré-Cartan $(m+1)$-form may be written as

$$
\Omega_{\mathcal{L}}=\omega \wedge\left(\gamma_{\mathcal{L}}\right)_{\eta}^{\nabla}+\Omega_{\mathcal{L}}^{\nabla}
$$

where $\left(\gamma_{\mathcal{L}}\right)_{\eta}^{\nabla}=i\left(\mathcal{Y}_{\eta}^{\nabla}\right) \Omega_{\mathcal{L}} \in \Omega^{1}\left(J^{1} \pi\right)$, and $\Omega_{\mathcal{L}}^{\nabla}$ is a $(m+1)$-form on $J^{1} \pi$ of bidegree $(m-1,2)$ with respect to the connection $\nabla$.

( Proof) If $y \in J^{1} \pi$ and $v_{1}, v_{2}, v_{3} \in V_{y}\left(\bar{\pi}^{1}\right)\left(V\left(\bar{\pi}^{1}\right)\right.$ being the vertical bundle of $\bar{\pi}^{1}$ ) then, from (18) we have that $i\left(v_{1} \wedge v_{2} \wedge v_{3}\right) \Omega_{\mathcal{L}}(y)=0$. Thus, the result follows from Propositions 2 and 5 .

If $\left(J^{1} \pi, \Omega_{\mathcal{L}}\right)$ is a hyperregular Lagrangian system (the regular case is analogous) $\mathcal{F} \mathcal{L}$ is a global diffeomorphism. Moreover, if $\Omega_{h}$ is the Hamilton-Cartan $(m+1)$-form on $J^{1} \pi^{*}$, then

$$
\mathcal{F} \mathcal{L}^{*} \Omega_{h}=\Omega_{\mathcal{L}}
$$

Furthermore, as $\mathcal{F} \mathcal{L}$ is a global diffeomorphism, the connection $\nabla$ induces a connection $\nabla^{*}$ in the fibration $\bar{\tau}^{1}: J^{1} \pi^{*} \rightarrow M$ in such a way that

$$
\mathcal{F} \mathcal{L}_{*} \mathcal{Y}_{\eta}^{\nabla}=\mathcal{Y}_{\eta}^{\nabla^{*}}
$$

where $\mathcal{Y}_{\eta}^{\nabla^{*}}$ is the $m$-vector field on $J^{1} \pi^{*}$ associated with $\nabla^{*}$ and the volume form $\eta$. Thus, from (19), (201) and Proposition [7, we obtain:

Proposition 8 The Hamilton-Cartan $(m+1)$-form may be written as

$$
\Omega_{h}=\Omega_{h}^{\nabla^{*}}+\omega^{*} \wedge\left(\gamma_{h}\right)_{\eta}^{\nabla^{*}}
$$

where $\left(\gamma_{h}\right)_{\eta}^{\nabla^{*}}=i\left(\mathcal{Y}_{\eta}^{\nabla^{*}}\right) \Omega_{h}$, and $\Omega_{h}^{\nabla^{*}}$ is a $(m+1)$-form on $J^{1} \pi^{*}$ of bidegree $(m-1,2)$ with respect to the connection $\nabla^{*}$.

Furthermore, we may prove the following result:

Proposition 9 If $\left(J^{1} \pi, \Omega_{\mathcal{L}}\right)$ is a regular Lagrangian system, then the $(m+1)$-forms $\Omega_{\mathcal{L}}^{\nabla}$ and $\Omega_{h}^{\nabla^{*}}$ are 1-nondegenerate.

( Proof ) As $\mathcal{F} \mathcal{L}$ is a diffeomorphism and $\mathcal{F} \mathcal{L}^{*} \Omega_{h}^{\nabla^{*}}=\Omega_{\mathcal{L}}^{\nabla}$, it suffices to prove that $\Omega_{h}^{\nabla^{*}}$ is 1nondegenerate. The local expression of $\Omega_{h}^{\nabla^{*}}$ is

$$
\Omega_{h}^{\nabla^{*}}=-d p_{A}^{\alpha} \wedge d y^{A} \wedge d^{m-1} x_{\alpha}+\theta \wedge d^{m} x
$$

$\theta$ being a 1-form such that $\theta\left(\frac{\partial}{\partial x_{\alpha}}\right)=0$, for every $\alpha$. As a consequence,

$i\left(\frac{\partial}{\partial x_{\beta}}\right) \Omega_{h}^{\nabla^{*}}=-\sum_{A, \alpha ; \alpha \neq \beta} d p_{A}^{\alpha} \wedge d y^{A} \wedge d^{m-2} x_{\alpha \beta}-\theta\left(\frac{\partial}{\partial y^{A}}\right) d y^{A} \wedge d^{m-1} x_{\beta}-\theta\left(\frac{\partial}{\partial p_{A}^{\alpha}}\right) d p_{A}^{\alpha} \wedge d^{m-1} x_{\beta}$ 


$$
\begin{gathered}
i\left(\frac{\partial}{\partial y^{A}}\right) \Omega_{h}^{\nabla^{*}}=\sum_{\alpha} d p_{A}^{\alpha} \wedge d^{m-1} x_{\alpha}+\theta\left(\frac{\partial}{\partial y^{A}}\right) d^{m} x \\
i\left(\frac{\partial}{\partial p_{A}^{\alpha}}\right) \Omega_{h}^{\nabla^{*}}=-d y^{A} \wedge d^{m-1} x_{\alpha}+\theta\left(\frac{\partial}{\partial p_{A}^{\alpha}}\right) d^{m} x .
\end{gathered}
$$

Thus, if $X=\lambda_{\beta} \frac{\partial}{\partial x_{\beta}}+\mu^{A} \frac{\partial}{\partial y^{A}}+\nu_{A}^{\alpha} \frac{\partial}{\partial p_{A}^{\alpha}}$ is a local vector field such that $i(X) \Omega_{h}^{\nabla^{*}}=0$ then, from (21), it follows that $\lambda_{\beta}=0$, for every $\beta$, which implies that (see (22) and (23))

$$
\mu^{A} d p_{A}^{\alpha} \wedge d^{m-1} x_{\alpha}+\mu^{A} \theta\left(\frac{\partial}{\partial y^{A}}\right) d^{m} x-\nu_{A}^{\alpha} d y^{A} \wedge d^{m-1} x_{\alpha}+\nu_{\alpha}^{A} \theta\left(\frac{\partial}{\partial p_{A}^{\alpha}}\right) d^{m} x=0 .
$$

Therefore, $\mu^{A}=0$ and $\nu_{A}^{\alpha}=0$, for every $A$ and $\alpha$, that is, $X=0$.

If the Lagrangian is regular, then from Propositions [ 6 and 9 we obtain that $\left(T_{y}^{\perp} J^{1} \pi\right)_{\Omega_{\mathcal{L}}}^{\nabla}=\{0\}$, for every $y \in J^{1} \pi$. Thus, there exist locally decomposable $m$-vector fields $\mathcal{X}_{\mathcal{L}}$ on $J^{1} \pi$ such that

$$
i\left(\mathcal{X}_{\mathcal{L}}\right) \omega=1, \quad i\left(\mathcal{X}_{\mathcal{L}}\right) \Omega_{\mathcal{L}}=0
$$

Moreover, we have

Proposition 10 If $\left(J^{1} \pi, \Omega_{\mathcal{L}}\right)$ is a regular Lagrangian system and $\mathcal{X}_{\mathcal{L}}$ is a locally decomposable $m$ vector field on $J^{1} \pi$ such that $i\left(\mathcal{X}_{\mathcal{L}}\right) \omega=1$ and $i\left(\mathcal{X}_{\mathcal{L}}\right) \Omega_{\mathcal{L}}=0$ then $\mathcal{X}_{\mathcal{L}}$ is an Euler-Lagrange $m$-vector field for $\mathcal{L}$.

( Proof) We must prove that $\mathcal{X}_{\mathcal{L}}$ is semi-holonomic, that is, $i\left(\mathcal{X}_{\mathcal{L}}\right) \mathcal{V}=0$. For this purpose, we consider local fibred coordinates $\left(x^{\alpha}, y^{A}, v_{\alpha}^{A}\right)$ on $J^{1} \pi$. Then, since $i\left(\mathcal{X}_{\mathcal{L}}\right) \omega=1$, it follows that

$$
\mathcal{X}_{\mathcal{L}}=\Lambda_{\alpha=1}^{m}\left(\frac{\partial}{\partial x^{\alpha}}+\Gamma_{\alpha}^{A} \frac{\partial}{\partial y^{A}}+\Gamma_{\alpha \beta}^{A} \frac{\partial}{\partial v_{\beta}^{A}}\right)
$$

with $\Gamma_{\alpha}^{A}$ and $\Gamma_{\alpha \beta}^{A}$ local real functions on $J^{1} \pi$. Furthermore, from (18), we deduce that

$$
\begin{aligned}
& 0=\left(i\left(\mathcal{X}_{\mathcal{L}}\right) \Omega_{\mathcal{L}}\right)\left(\frac{\partial}{\partial v_{\nu}^{B}}\right)=(-1)^{m} i\left(\mathcal{X}_{\mathcal{L}}\right)\left(i\left(\frac{\partial}{\partial v_{\nu}^{B}}\right) \Omega_{\mathcal{L}}\right) \\
& =(-1)^{m+1}\left(\Gamma_{\alpha}^{A}-v_{\alpha}^{A}\right) \frac{\partial^{2} L}{\partial v_{\alpha}^{A} \partial v_{\nu}^{B}}, \quad \text { for all } B \text { and } \nu .
\end{aligned}
$$

Therefore, using the fact that $\mathcal{L}$ is regular, we conclude that

$$
\Gamma_{\alpha}^{A}=v_{\alpha}^{A}, \quad \text { for all } A \text { and } \alpha,
$$

which implies that $\mathcal{X}_{\mathcal{L}}$ is semi-holonomic.

Hence, if $\left(J^{1} \pi, \Omega_{\mathcal{L}}\right)$ is a regular Lagrangian system, then the existence of classes of EulerLagrange $m$-vector fields for $\mathcal{L}$ is assured in $J^{1} \pi$. In the same way, for the Hamiltonian formalism, the existence of Hamilton-De Donder-Weyl $m$-vector fields is assured everywhere in $J^{1} \pi^{*}$ (note that if $\mathcal{X}_{\mathcal{L}}$ is an Euler-Lagrange $m$-vector field for $\mathcal{L}$ then $(\mathcal{F} \mathcal{L})_{*} \mathcal{X}_{\mathcal{L}}$ is a Hamilton-De Donder-Weyl $m$-vector field on $\left.J^{1} \pi^{*}\right)$. In both cases, the solution is not unique.

For singular (almost-regular) Lagrangian systems, the existence of Euler-Lagrange $m$-vector fields is not assured except perhaps on some submanifold $S_{f} \hookrightarrow J^{1} \pi$, where the solution is not 
unique. In order to find this submanifold we apply the algorithm developed in Section 3.3 to the system $\left(J^{1} \pi, \Omega_{\mathcal{L}}\right)$, by doing the identifications $\kappa: F \rightarrow M$ with $\bar{\pi}^{1}: J^{1} \pi \rightarrow M$, and $\Omega$ with $\Omega_{\mathcal{L}}$. Thus we obtain obtain a sequence

$$
\cdots \stackrel{j_{i+1}^{i}}{\hookrightarrow} N_{i} \stackrel{j_{i}^{i-1}}{\hookrightarrow} \cdots \stackrel{j_{2}^{1}}{\hookrightarrow} N_{1} \stackrel{j_{1}}{\hookrightarrow} N_{0} \equiv J^{1} \pi
$$

which, in the best of cases stabilizes in the final constraint submanifold $N_{f}$ where there exist $m$-vector fields $\mathcal{X}^{N_{f}}$ on $N_{f}$, solution to the equations

$$
\left.\left(i\left(\mathcal{X}^{N_{f}}\right) \Omega_{\mathcal{L}}\right)\right|_{N_{f}}=0,\left.\quad\left(i\left(\mathcal{X}^{N_{f}}\right) \omega\right)\right|_{N_{f}}=1 .
$$

But $\mathcal{X}^{N_{f}}$ will not be, in general, an Euler-Lagrange $m$-vector field on $N_{f}$ (that is, it is not semiholonomic), and, in addition, $\mathcal{X}^{N_{f}}$ will not in general be an integrable $m$-vector field. The problem of finding integrable Euler-Lagrange $m$-vector fields (i.e., holonomic) is discussed and solved in the next Section.

Now, we consider the Hamiltonian system $\left(\mathcal{P}, \Omega_{\mathrm{h}}^{0}\right)$. Let $\nabla_{0}^{*}$ be a connection in the bundle $\bar{\tau}_{0}^{1}: \mathcal{P} \rightarrow M$ and denote by $\mathcal{Y}_{\eta}^{\nabla_{0}^{*}}$ the corresponding $m$-vector field on $\mathcal{P}$ associated with $\nabla_{0}^{*}$ and $\eta$. Then, we have:

Proposition 11 The Hamilton-Cartan $(m+1)$-form may be written as

$$
\Omega_{h}^{0}=\Omega_{h}^{\nabla_{0}^{*}}+\omega_{0}^{*} \wedge\left(\gamma_{h}\right)_{\eta}^{\nabla_{0}^{*}}
$$

where $\omega_{0}^{*}=\bar{\tau}_{0}^{1 *} \eta,\left(\gamma_{h}\right)_{\eta}^{\nabla_{0}^{*}}=i\left(\mathcal{Y}_{\eta}^{\nabla_{0}^{*}}\right) \Omega_{h}$, and $\Omega_{h}^{\nabla_{0}^{*}}$ is a $(m+1)$-form on $\mathcal{P}$ of bidegree $(m-1,2)$ with respect to the connection $\nabla_{0}^{*}$.

( Proof) If $\bar{y}=\mathcal{F} \mathcal{L}_{0}(y) \in \mathcal{P}$, with $y \in J^{1} \pi$, and $\bar{v}_{1}, \bar{v}_{2}, \bar{v}_{3} \in V_{y}\left(\bar{\tau}_{0}^{1}\right)$ then, since $\mathcal{F} \mathcal{L}_{0}: J^{1} \pi \rightarrow \mathcal{P}$ is a submersion and $\bar{\tau}_{0}^{1} \circ \mathcal{F} \mathcal{L}_{0}=\bar{\pi}^{1}$, it follows that there exist $v_{1}, v_{2}, v_{3} \in V_{y}\left(\bar{\pi}^{1}\right)$ such that

$$
\left(T_{y} \mathcal{F} \mathcal{L}_{0}\right)\left(v_{i}\right)=\bar{v}_{i}, \quad \text { for } i \in\{1,2,3\}
$$

Thus, using that $\left(\mathcal{F} \mathcal{L}_{0}\right)^{*} \Omega_{h}^{0}=\Omega_{\mathcal{L}}$, we deduce that

$$
i\left(\bar{v}_{1} \wedge \bar{v}_{2} \wedge \bar{v}_{3}\right) \Omega_{h}^{0}(\bar{y})=0
$$

This proves the result.

Hamilton-De Donder-Weyl $m$-vector fields do not exist, in general, in $\mathcal{P}$, and then we must apply the algorithmic procedure developed in Section 3.3 to the system $\left(\mathcal{P}, \Omega_{\mathrm{h}}^{0}\right)$, by doing the identifications $\kappa: F \rightarrow M$ with $\left.\bar{\tau}^{1}\right|_{\mathcal{P}}: \mathcal{P} \rightarrow M$, and $\Omega$ with $\Omega_{h}^{0}$. Thus we obtain a sequence

$$
\cdots \stackrel{j_{i+1}^{i}}{\longrightarrow} P_{i} \stackrel{j_{i}^{i-1}}{\longrightarrow} \cdots \stackrel{j_{2}^{1}}{\longrightarrow} P_{1} \stackrel{j_{1}}{\longrightarrow} P_{0} \equiv \mathcal{P}
$$

which, in the best of cases stabilizes in the final constraint submanifold $P_{f}$ of $\mathcal{P}$ where there exist $m$-vector fields $\mathcal{X}^{P_{f}}$ on $P_{f}$, solution to the equations

$$
\left.\left(i\left(\mathcal{X}^{P_{f}}\right) \Omega_{h}^{0}\right)\right|_{P_{f}}=0,\left.\quad\left(i\left(\mathcal{X}^{P_{f}}\right) \omega_{0}^{*}\right)\right|_{P_{f}}=1 .
$$

Of course the solution $\mathcal{X}^{P_{f}}$ is not unique. 
Remark 3 The Lagrangian and Hamiltonian pre-multisymplectic algorithms are equivalent in the following sense: at every level $j$ of the Lagrangian and Hamiltonian algorithms, the submanifolds of the sequences (24) and (26) are $\mathcal{F} \mathcal{L}$-related, that is, $\mathcal{F} \mathcal{L}\left(N_{j}\right)=P_{j}$ and $\mathcal{F} \mathcal{L}_{j}=\mathcal{F} \mathcal{L}_{\mid N_{j}}: N_{j} \rightarrow P_{j}$ is a submersion such that $\mathcal{F} \mathcal{L}_{j}^{-1}\left(\mathcal{F} \mathcal{L}_{j}\left(x_{j}\right)\right)=\mathcal{F} \mathcal{L}_{0}^{-1}\left(\mathcal{F} \mathcal{L}_{0}\left(x_{j}\right)\right)$, for $x_{j} \in N_{j}$. Moreover, if $N_{f}$ is the final constraint submanifold (in the Lagrangian level) and $\mathcal{X}^{N_{f}}$ is a locally decomposable $m$ vector field on $N_{f}$ such that equations (25) hold and, in addition, $\mathcal{X}^{N_{f}}$ is $\mathcal{F} \mathcal{L}_{f}$-projectable to an $m$-vector field $\mathcal{X}^{P_{f}}$ on $P_{f}$ then $\mathcal{X}^{P_{f}}$ is locally decomposable and equations (27) hold. Conversely, if $\mathcal{X}^{P_{f}}$ is a locally decomposable $m$-vector field on $P_{f}$ satisfying equations (27) and $\mathcal{X}^{N_{f}}$ is a locally decomposable $m$-vector field on $N_{f}$ which is $\mathcal{F} \mathcal{L}_{f}$-projectable on $\mathcal{X}^{P_{f}}$ then $\mathcal{X}^{N_{f}}$ satisfies equations (25) (see [26, 30] for a detailed discussion on this topic).

Finally, the Hamilton-De Donder-Weyl $m$-vector fields $\mathcal{X}^{P_{f}}$ are not integrable, in general. In fact, if we have that $\mathcal{X}^{P_{f}}=X_{1}^{P_{f}} \wedge \ldots \wedge X_{m}^{P_{f}}$, where $X_{\alpha}^{P_{f}}$ are (local) vector fields on $P_{f}$, for all $\alpha$, and $\left\{X_{1}^{P_{f}}, \ldots, X_{m}^{P_{f}}, \bar{Z}_{1}, \ldots, \bar{Z}_{p}\right\}$ is a local basis of the vector bundle $T P_{f} \rightarrow P_{f}$ then

$$
\left[X_{\alpha}^{P_{f}}, X_{\beta}^{P_{f}}\right]=\bar{f}_{\alpha \beta}^{\gamma} X_{\gamma}^{P_{f}}+\bar{\zeta}_{\alpha \beta}^{l} \bar{Z}_{l}
$$

for some functions $\bar{f}_{\alpha \beta}^{\gamma}$ and $\bar{\zeta}_{\alpha \beta}^{l}$ on $P_{f}$. Therefore, we must apply the integrability algorithm of Section 3.4 and we obtain a sequence $\ldots \subseteq \mathcal{J}_{i} \subseteq \ldots \subseteq \mathcal{J}_{1} \subseteq P_{f}$, such that $\mathcal{J}_{i}$ is a non-empty (closed) submanifold of $S_{f}$, with

$$
\begin{aligned}
\mathcal{J}_{1} & =\left\{y \in P_{f} \mid \bar{\zeta}_{\alpha \beta}^{l}(y)=0\right\} \\
\mathcal{J}_{i} & =\left\{y \in \mathcal{J}_{i-1} \mid \mathcal{X}^{P_{f}}(y) \in \Lambda^{m} \mathrm{~T}_{y} \mathcal{J}_{i-1}\right\}, \text { for } i \geq 2 .
\end{aligned}
$$

In the best cases, there exists an integer $i$ such that $\mathcal{J}_{i+1}=\mathcal{J}_{i}$. Then, $\mathcal{J}_{f}=\mathcal{J}_{i+1}=\mathcal{J}_{i}$ is a submanifold of $P_{f}$, and $\mathcal{X}^{\mathcal{J}_{f}}=\left.\left(\mathcal{X}^{P_{f}}\right)\right|_{\mathcal{J}_{f}}$ is an integrable Hamilton-De Donder-Weyl $m$-vector field in $\mathcal{J}_{f}$.

\subsection{Almost-regular Lagrangians and integrable Euler-Lagrange $m$-vector fields}

Let $\left(J^{1} \pi, \Omega_{\mathcal{L}}\right)$ be an almost-regular Lagrangian system, and $N_{f}$ the final constraint submanifold (in the Lagrangian setting). Then, there exists a locally decomposable $m$-vector field $\mathcal{X}^{N_{f}}$ on $N_{f}$ such that

$$
\left.\left(i\left(\mathcal{X}^{N_{f}}\right) \Omega_{\mathcal{L}}\right)\right|_{N_{f}}=0,\left.\quad\left(i\left(\mathcal{X}^{N_{f}}\right) \omega\right)\right|_{N_{f}}=1 .
$$

But, in general, $\mathcal{X}^{N_{f}}$ is not an Euler-Lagrange $m$-vector field on $N_{f}$ and, in addition, $\mathcal{X}^{N_{f}}$ will not in general be an integrable $m$-vector field.

In order to solve these problems, first we construct a submanifold $S_{f}$ of $N_{f}$ where there exists a locally decomposable $m$-vector field $\mathcal{X}^{S_{f}}$ such that

$$
\left.\left(i\left(\mathcal{X}^{S_{f}}\right) \Omega_{\mathcal{L}}\right)\right|_{S_{f}}=0,\left.\quad\left(i\left(\mathcal{X}^{S_{f}}\right) \omega\right)\right|_{S_{f}}=1,\left.\quad\left(i\left(\mathcal{X}^{S_{f}}\right) \mathcal{V}\right)\right|_{S_{f}}=0 .
$$

In fact, from the above discussion we know that we can choose the $m$-vector field $\mathcal{X}^{N_{f}}$ on $N_{f}$ such that it projects via $\mathcal{F} \mathcal{L}_{f}$ (the restriction of $\mathcal{F} \mathcal{L}$ to $N_{f}$ ) onto an $m$-vector field $\mathcal{X}^{P_{f}}$ on $P_{f}$. Then, we consider the subset $S_{f}$ of $N_{f}$ defined by

$$
S_{f}=\left\{x \in N_{f} /\left(i\left(\mathcal{X}^{N_{f}}\right) \mathcal{V}\right)(x)=0\right\} .
$$


In [30] (see also 26]), it was proved that

$$
\left(i\left(\mathcal{X}^{N_{f}}\right) \mathcal{V}\right)(x) \in \operatorname{Ker} \mathrm{T}_{x}\left(\mathcal{F} \mathcal{L}_{f}\right)=\operatorname{Ker} \mathrm{T}_{x}\left(\mathcal{F} \mathcal{L}_{0}\right)
$$

and that for every $x \in N_{f}, S_{f} \cap \mathcal{F} \mathcal{L}_{f}^{-1}\left(\mathcal{F} \mathcal{L}_{f}(x)\right)=S_{f} \cap \mathcal{F} \mathcal{L}_{0}^{-1}\left(\mathcal{F} \mathcal{L}_{0}(x)\right)$ is a single point in $S_{f}$.

The above result allows us to introduce a well-defined map $s_{f}: P_{f} \rightarrow N_{f}$ such that

$$
S_{f}=s_{f}\left(P_{f}\right), \quad \mathcal{F} \mathcal{L}_{f} \circ s_{f}=I d .
$$

Thus, $s_{f}: P_{f} \rightarrow N_{f}$ is a global section of the submersion $\mathcal{F} \mathcal{L}_{f}: N_{f} \rightarrow P_{f}$ and, therefore, $S_{f}$ is an embedded submanifold of $N_{f}$ and the map $s_{f}: P_{f} \rightarrow S_{f}$ is a diffeomorphism (for more details, see [26, 30].

Now, defining the $m$-vector field $\mathcal{X}^{S_{f}}$ on $S_{f}$ by $\mathcal{X}^{S_{f}}=\left(\Lambda^{m} \mathrm{~T} s_{f}\right) \circ \mathcal{X}^{P_{f}}$, then we have [30]:

Theorem $6 \mathcal{X}^{S_{f}}$ is an Euler-Lagrange m-vector field on $S_{f}$ for the Lagrangian $\mathcal{L}$, that is, $\mathcal{X}^{S_{f}}$ is a locally decomposable m-vector field on $S_{f}$ and

$$
\left.\left(i\left(\mathcal{X}^{S_{f}}\right) \Omega_{\mathcal{L}}\right)\right|_{S_{f}}=0,\left.\quad\left(i\left(\mathcal{X}^{S_{f}}\right) \omega\right)\right|_{S_{f}}=1,\left.\quad\left(i\left(\mathcal{X}^{S_{f}}\right) \mathcal{V}\right)\right|_{S_{f}}=0
$$

Next, we give a local description of the submanifold $S_{f}$ and of the Euler-Lagrange $m$-vector field $\mathcal{X}^{S_{f}}$ on $S_{f}$. Since $\mathcal{L}$ is almost-regular, it follows that the rank of the partial Hessian matrix $\left(\frac{\partial^{2} L}{\partial v_{\alpha}^{A} \partial v_{\beta}^{B}}\right)$ is constant. Let $\operatorname{rank}\left(\frac{\partial^{2} L}{\partial v_{\alpha}^{A} \partial v_{\beta}^{B}}\right)=p m+q$, with $0 \leq p \leq n-1$ and $0 \leq q \leq m$, and assume that the first $p m+q$ rows of this matrix are independent. Denote by $\widetilde{V\left(\pi^{1}\right)} \rightarrow J^{1} \pi$ the vector subbundle of the vertical bundle $V\left(\pi^{1}\right) \rightarrow J^{1} \pi$ of $\pi^{1}: J^{1} \pi \rightarrow M$ generated by the local vector fields

$$
\left\{\frac{\partial}{\partial v_{\alpha}^{A}}, \frac{\partial}{\partial v_{1}^{p+1}}, \ldots, \frac{\partial}{\partial v_{q}^{p+1}}\right\}, \quad \text { for } 1 \leq A \leq p \text { and } 1 \leq \alpha \leq m .
$$

Then, there exist sections $\left\{X_{q+1}^{p+1}, \ldots, X_{m}^{p+1}, X_{\alpha}^{A}\right\}$, with $p+2 \leq A \leq n$ and $1 \leq \alpha \leq m$, of the vector bundle $\widetilde{V\left(\pi^{1}\right)} \rightarrow J^{1} \pi$ such that $\left\{W_{q+1}^{p+1}, \ldots, W_{m}^{p+1}, W_{\alpha}^{A}\right\}$, with $p+2 \leq A \leq n$ and $1 \leq \alpha \leq m$, is a local basis of $\operatorname{Ker}\left(\mathrm{T}\left(\mathcal{F} \mathcal{L}_{0}\right)\right)$, where

$$
W_{\beta}^{p+1}=\frac{\partial}{\partial v_{\beta}^{p+1}}+X_{\beta}^{p+1}, \quad W_{\alpha}^{A}=\frac{\partial}{\partial v_{\alpha}^{A}}+X_{\alpha}^{A}, \quad(p+2 \leq A \leq n, 1 \leq \alpha \leq m, q+1 \leq \beta \leq m) .
$$

Now, suppose that $\mathcal{X}^{N_{f}}=\mathcal{X}_{1}^{N_{f}} \wedge \ldots \wedge \mathcal{X}_{m}^{N_{f}}$, with

$$
\mathcal{X}_{\alpha}^{N_{f}}=\left.\left(\frac{\partial}{\partial x^{\alpha}}+\Gamma_{\alpha}^{A} \frac{\partial}{\partial y^{A}}+\Gamma_{\alpha \beta}^{A} \frac{\partial}{\partial v_{\beta}^{A}}\right)\right|_{N_{f}}, \text { for } \alpha \in\{1, \ldots, m\} \text {. }
$$

Then, using that $\mathcal{X}^{N_{f}}$ is $\mathcal{F} \mathcal{L}_{f}$-projectable, it follows that the functions $\Gamma_{\alpha}^{A}$ are constant on the fibers of $\mathcal{F} \mathcal{L}_{f}: N_{f} \rightarrow P_{f}$. But, as $\mathcal{F} \mathcal{L}_{f}^{-1}\left(\mathcal{F} \mathcal{L}_{f}(x)\right)=\mathcal{F} \mathcal{L}_{0}^{-1}\left(\mathcal{F} \mathcal{L}_{0}(x)\right.$ ), for every $x \in N_{f}$ (see Remark 3i), we obtain that

$$
\begin{array}{lr}
W_{\gamma}^{p+1}\left(\Gamma_{\alpha}^{A}\right)=0, & \gamma \in\{q+1, \ldots, m\} \\
W_{\gamma}^{p+1+i}\left(\Gamma_{\alpha}^{A}\right)=0, \quad i \in\{1, \ldots, n-p-1\}, \quad \gamma \in\{1, \ldots, m\} .
\end{array}
$$

Furthermore

$$
i\left(\mathcal{X}^{N_{f}}\right) \mathcal{V}=\left(\Gamma_{\alpha}^{A}-v_{\alpha}^{A}\right) \frac{\partial}{\partial v_{\alpha}^{A}}
$$


Thus, from (29) and (30), we have that

$$
i\left(\mathcal{X}^{N_{f}}\right) \mathcal{V}=\left(\Gamma_{\gamma}^{p+1}-v_{\gamma}^{p+1}\right) W_{\gamma}^{p+1}+\left(\Gamma_{\gamma}^{p+1+i}-v_{\gamma}^{p+1+i}\right) W_{\gamma}^{p+1+i} .
$$

Note that the functions

$$
\begin{aligned}
& \zeta_{\gamma}^{p+1}=\Gamma_{\gamma}^{p+1}-v_{\gamma}^{p+1}, \quad \gamma \in\{q+1, \ldots, m\} \\
& \zeta_{\bar{\gamma}}^{p+1+i}=\Gamma_{\bar{\gamma}}^{p+1+i}-v_{\bar{\gamma}}^{p+1+i}, \quad i \in\{1, \ldots, n-p-1\}, \quad \bar{\gamma} \in\{1, \ldots, m\} .
\end{aligned}
$$

are independent on $N_{f}$. In fact (see (30), (31) and (33))

$$
\begin{array}{lr}
W_{\gamma}^{p+1}\left(\zeta_{\gamma^{\prime}}^{p+1}\right)=-\delta_{\gamma \gamma^{\prime}}, & W_{\gamma}^{p+1}\left(\zeta_{\bar{\gamma}}^{p+1+i}\right)=0 \\
W_{\bar{\gamma}}^{p+1+i}\left(\zeta_{\gamma}^{p+1}\right)=0, & W_{\bar{\alpha}}^{p+1+i}\left(\zeta_{\bar{\gamma}}^{p+1+j}\right)=-\delta_{i j} \delta_{\bar{\alpha} \bar{\gamma}}
\end{array}
$$

Moreover, using (28) and (32), we conclude that $\left\{\zeta_{\gamma}^{p+1}, \zeta_{\bar{\gamma}}^{p+1+i}\right\}$, with $\gamma \in\{1, \ldots, m\}, i \in\{1, \ldots, n-$ $p-1\}$ and $\bar{\gamma} \in\{1, \ldots, m\}$, is a set of local independent constraint functions defining $S_{f}$ as a submanifold of $N_{f}$, that is,

$$
S_{f}=\left\{x \in N_{f} /\left(\Gamma_{\gamma}^{p+1}-v_{\gamma}^{p+1}\right)(x)=0, \quad\left(\Gamma_{\bar{\gamma}}^{p+1+i}-v_{\bar{\gamma}}^{p+1+i}\right)(x)=0\right\} .
$$

Finally, a direct calculation proves that the Euler-Lagrange $m$-vector field $\mathcal{X}^{S_{f}}$ on $S_{f}$ is given by $\mathcal{X}^{S_{f}}=\mathcal{X}_{1}^{S_{f}} \wedge \ldots \wedge \mathcal{X}_{m}^{S_{f}}$, with

$$
\mathcal{X}_{\alpha}^{S_{f}}=\left.\left(\mathcal{X}_{\alpha}^{N_{f}}+\mathcal{X}_{\alpha}^{N_{f}}\left(\zeta_{\gamma}^{p+1}\right) W_{\gamma}^{p+1}+\mathcal{X}_{\alpha}^{N_{f}}\left(\zeta_{\bar{\gamma}}^{p+1+i}\right) W_{\bar{\gamma}}^{p+1+i}\right)\right|_{S_{f}}, \quad \text { for every } \alpha .
$$

$\mathcal{X}^{S_{f}}$ is not, in general, integrable. In fact, if $\left\{\mathcal{X}_{1}^{S_{f}}, \ldots, \mathcal{X}_{m}^{S_{f}}, Z_{1}, \ldots, Z_{s}\right\}$ is a local basis of the vector bundle $T S_{f} \rightarrow S_{f}$ then we have that

$$
\left[\mathcal{X}_{\alpha}^{S_{f}}, \mathcal{X}_{\beta}^{S_{f}}\right]=f_{\alpha \beta}^{\gamma} \mathcal{X}_{\gamma}^{S_{f}}+\zeta_{\alpha \beta}^{l} Z_{l}
$$

for some functions $f_{\alpha \beta}^{\gamma}$ and $\zeta_{\alpha \beta}^{l}$.

Therefore, we must apply the integrability algorithm of Section 3.4 Then, we obtain a sequence $\ldots \subseteq \mathcal{I}_{i} \subseteq \ldots \subseteq \mathcal{I}_{1} \subseteq S_{f}$, such that $\mathcal{I}_{i}$ is a non-empty (closed) submanifold of $S_{f}$, with

$$
\begin{aligned}
\mathcal{I}_{1} & =\left\{x \in S_{f} \mid \zeta_{\alpha \beta}^{\gamma}(x)=0\right\} \\
\mathcal{I}_{i} & =\left\{x \in \mathcal{I}_{i-1} \mid \mathcal{X}^{S_{f}}(x) \in \Lambda^{m} \mathrm{~T}_{x} \mathcal{I}_{i-1}\right\}, \text { for } i \geq 2
\end{aligned}
$$

In the best cases, there exists an integer $i$ such that $\mathcal{I}_{i+1}=\mathcal{I}_{i}$. Then, $\mathcal{I}_{f}=\mathcal{I}_{i+1}=\mathcal{I}_{i}$ is a submanifold of $S_{f}$ and $\mathcal{X}^{\mathcal{I}_{f}}=\left.\left(\mathcal{X}^{S_{f}}\right)\right|_{\mathcal{I}_{f}}$ is an integrable Euler-Lagrange $m$-vector field on $\mathcal{I}_{f}$, and hence it is holonomic. In fact:

Theorem 7 If $U$ is an open subset of $M$ and $s: U \subseteq M \rightarrow \mathcal{I}_{f}$ is an integral section of $\mathcal{X}^{\mathcal{I}_{f}}$ then there exists a section $\phi: U \subseteq M \rightarrow E$ of the projection $\pi: E \rightarrow M$ such that $s=j^{1} \phi$ and $\phi$ is a solution to the Euler-Lagrange equations for $\mathcal{L}$.

( Proof ) We have that

$$
\begin{aligned}
& \left.\left(i\left(\mathcal{X}^{f}\right) \Omega_{\mathcal{L}}\right)\right|_{\mathcal{I}_{f}}=0, \\
& \left.\left(i\left(\mathcal{X}^{f}\right) \mathcal{V}\right)\right|_{\mathcal{I}_{f}}=0 .
\end{aligned}
$$


We can assume, without loss of generality, that $s(U) \subseteq \tilde{U}$, with $\tilde{U}$ an open subset of $J^{1} \pi$ and $\left(x^{\alpha}, y^{A}, v_{\alpha}^{A}\right)$ a system of local coordinates on $\tilde{U}$. Then, since $\mathcal{X}^{\mathcal{I}_{f}}$ is locally decomposable and $\left.i\left(\mathcal{X}^{f}\right) \omega\right|_{\mathcal{I}_{f}}=1$, we deduce that

$$
\left.\mathcal{X}^{\mathcal{I}_{f}}\right|_{\tilde{U} \cap \mathcal{I}_{f}}=\mathcal{X}_{1}^{\mathcal{I}_{f}} \wedge \ldots \wedge \mathcal{X}_{m}^{\mathcal{I}_{f}}
$$

with $\mathcal{X}_{\alpha}^{\mathcal{I}_{f}} \in \mathfrak{X}\left(\tilde{U} \cap \mathcal{I}_{f}\right)$ given by

$$
\mathcal{X}_{\alpha}^{\mathcal{I}_{f}}=\left.\left(\frac{\partial}{\partial x^{\alpha}}+\Gamma_{\alpha}^{A} \frac{\partial}{\partial y^{A}}+\Gamma_{\alpha \beta}^{A} \frac{\partial}{\partial v_{\beta}^{A}}\right)\right|_{\tilde{U} \cap \mathcal{I}_{f}}
$$

for all $\alpha$, where $\Gamma_{\alpha}^{A}$ and $\Gamma_{\alpha \beta}^{A}$ are local functions on $\tilde{U}$. Now, using that

$$
\left.\left(i\left(\mathcal{X}^{\mathcal{I}_{f}}\right) \mathcal{V}\right)\right|_{\tilde{U} \cap \mathcal{I}_{f}}=\left.\left(\left(\Gamma_{\alpha}^{A}-v_{\alpha}^{A}\right) \frac{\partial}{\partial v_{\alpha}^{A}}\right)\right|_{\tilde{U} \cap \mathcal{I}_{f}}
$$

it follows that (see (35))

$$
\mathcal{X}_{\alpha}^{\mathcal{I}_{f}}=\left.\left(\frac{\partial}{\partial x^{\alpha}}+v_{\alpha}^{A} \frac{\partial}{\partial y^{A}}+\Gamma_{\alpha \beta}^{A} \frac{\partial}{\partial v_{\beta}^{A}}\right)\right|_{\tilde{U} \cap \mathcal{I}_{f}} .
$$

Furthermore, from (18), we obtain that

$$
\begin{aligned}
i\left(\frac{\partial}{\partial y^{A}}\right) \Omega_{\mathcal{L}}= & \frac{\partial^{2} L}{\partial v_{\nu}^{B} \partial v_{\alpha}^{A}} \mathrm{~d} v_{\nu}^{B} \wedge \mathrm{d}^{m-1} x^{\alpha}+\left(\frac{\partial^{2} L}{\partial y^{B} \partial v_{\alpha}^{A}}-\frac{\partial^{2} L}{\partial y^{A} \partial v_{\alpha}^{B}}\right) \mathrm{d} y^{B} \wedge \mathrm{d}^{m-1} x^{\alpha} \\
& +\left(\frac{\partial^{2} L}{\partial y^{A} \partial v_{\alpha}^{B}} v_{\alpha}^{B}-\frac{\partial L}{\partial y^{A}}+\frac{\partial^{2} L}{\partial x^{\alpha} \partial v_{\alpha}^{A}}\right) \mathrm{d}^{m} x
\end{aligned}
$$

Therefore, using (34), (36), (37) and (39), we conclude that

$$
\frac{\partial^{2} L}{\partial x^{\alpha} \partial v_{\alpha}^{A}}+\frac{\partial^{2} L}{\partial y^{B} \partial v_{\alpha}^{A}} v_{\alpha}^{B}+\frac{\partial^{2} L}{\partial v_{\nu}^{B} \partial v_{\alpha}^{A}} \Gamma_{\alpha \nu}^{B}-\frac{\partial L}{\partial y^{A}}=0, \text { for every } A .
$$

Next, suppose that $U$ is an open subset of $M$ and that $s: U \subseteq M \rightarrow \tilde{U} \cap \mathcal{I}_{f} \subseteq J^{1} \pi$ is an integral section of $\left.\mathcal{X}^{\mathcal{I}_{f}}\right|_{\tilde{U} \cap \mathcal{I}_{f}}$ such that the local expression of $s$ is $s\left(x^{\beta}\right)=\left(x^{\beta}, s^{A}\left(x^{\beta}\right), s_{\alpha}^{A}\left(x^{\beta}\right)\right)$. Using (388) and the fact that

$$
(\mathrm{T} s)\left(\frac{\partial}{\partial x^{\beta}}\right)=\left.\left(\frac{\partial}{\partial x^{\beta}}+\frac{\partial s^{A}}{\partial x^{\beta}} \frac{\partial}{\partial y^{A}}+\frac{\partial s_{\alpha}^{A}}{\partial x^{\beta}} \frac{\partial}{\partial v_{\beta}^{A}}\right)\right|_{\tilde{U} \cap \mathcal{I}_{f}}, \text { for every } \beta .
$$

we deduce that

$$
s_{\alpha}^{A}=\frac{\partial s^{A}}{\partial x^{\alpha}}, \quad \Gamma_{\alpha \beta}^{A} \circ s=\frac{\partial^{2} s^{A}}{\partial x^{\alpha} \partial x^{\beta}}, \text { for every } A, \alpha, \beta .
$$

From (41), it follows that there exists $\phi: U \subseteq M \rightarrow E$ a local section of $\pi: E \rightarrow M$ such that $s=j^{1} \phi$. Moreover, using (40) and (41), we obtain that

$$
\frac{\partial^{2} L}{\partial x^{\alpha} \partial v_{\alpha}^{A}}+\frac{\partial^{2} L}{\partial y^{B} \partial v_{\alpha}^{A}} \frac{\partial s^{B}}{\partial x^{\alpha}}+\frac{\partial^{2} L}{\partial v_{\nu}^{B} \partial v_{\alpha}^{A}} \frac{\partial^{2} s^{B}}{\partial x^{\alpha} \partial x^{\nu}}-\frac{\partial L}{\partial y^{A}}=0, \text { for every } A .
$$

This implies that

$$
\left(j^{i} \phi\right)^{*}\left(\frac{\partial L}{\partial y^{A}}-\frac{d}{d x^{\alpha}} \frac{\partial L}{\partial v_{\alpha}^{A}}\right)=0, \text { for every } A .
$$

In other words, $\phi$ is a solution to the Euler-Lagrange equations associated with $\mathcal{L}$. 
Remark 4 The behaviour of the integrability algorithm in the Lagrangian and Hamiltonian levels is the same. Indeed, it is easy to prove that $\left(\mathcal{F} \mathcal{L}_{f}\right)\left(\mathcal{I}_{i}\right)=\mathcal{J}_{i}$, and that the map $\left.\left(\mathcal{F} \mathcal{L}_{f}\right)\right|_{\mathcal{I}_{i}}: \mathcal{I}_{i} \rightarrow \mathcal{J}_{i}$ is a diffeomorphism, for every $i$. Thus, if the integrability algorithm in the Lagrangian level stabilizes at step $i$ then the integrability algorithm in the Hamiltonian level also stabilizes at step $i$ and, conversely, if the integrability algorithm in the Hamiltonian level stabilizes at step $i$ then the integrability algorithm in the Lagrangian level also stabilizes at step $i$.

\section{An example: affine Lagrangian densities}

Consider the configuration bundle $\pi: E \rightarrow M$, and $\alpha \in \Lambda_{1}^{m} \mathrm{~T}^{*} E$. Then, $\alpha$ induces a function $L=\hat{\alpha} \in \mathrm{C}^{\infty}\left(J^{1} \pi\right)$ as follows: given $x \in M$ and a section $\phi: M \rightarrow E$, we define $L\left(j_{x}^{1} \phi\right)$ by

$$
L\left(j_{x}^{1} \phi\right) \eta(x)=\left[\phi^{*} \alpha\right](x) .
$$

Note that $L\left(j_{x}^{1} \phi\right)$ is well-defined: if $\phi, \psi$ are sections such that $j_{x}^{1} \phi=j_{x}^{1} \psi$, then $L\left(j_{x}^{1} \phi\right)=L\left(j_{x}^{1} \psi\right)$. then

Taking fibered coordinates $\left(x^{\alpha}, y^{A}, v_{\alpha}^{A}\right)$ in $J^{1} E$, if $\alpha=a\left(x^{\alpha}, y^{A}\right) d^{m} x+f_{B}^{\mu}\left(x^{\alpha}, y^{A}\right) d y^{B} \wedge d^{m-1} x_{\mu}$,

$$
L\left(x^{\alpha}, y^{A}, v_{\alpha}^{A}\right)=a\left(x^{\alpha}, y^{A}\right)+f_{B}^{\mu}\left(x^{\alpha}, y^{A}\right) v_{\mu}^{B} .
$$

Thus, the Lagrangian density $\mathcal{L}=L \omega$ is affine.

A direct computation in local coordinates shows that $\Theta_{L}=\left(\pi^{1 *}\right) \alpha$ and, hence, $\Omega_{L}=(\pi)^{1 *}(-d \alpha)$. We also obtain $\widetilde{\mathcal{F} L}=\alpha \circ \pi^{1}$, and $\mathcal{F} \mathcal{L}=\mu \circ \alpha \circ \pi^{1}$. Therefore, $\tilde{\mathcal{P}}=\widetilde{\mathcal{F} L}\left(J^{1} \pi\right)=\alpha(E)$ is an embedded submanifold of $\mathcal{M} \pi$, which is diffeomorphic to $E$ by means of the mapping $\alpha: E \rightarrow \tilde{\mathcal{P}} \equiv \operatorname{Im} \alpha$. Since $\pi^{1}$ is a surjective submersion with connected fibers, then so is $\widetilde{\mathcal{F}}_{0}: J^{1} \pi \rightarrow \mathcal{P}$ (recall that $\widetilde{\mathcal{F}}_{0}$ is the restriction of $\widetilde{\mathcal{F} L}$ onto its image $\left.\mathcal{P}\right)$. Moreover, since $\widetilde{\mathcal{F} L}^{-1}(\widetilde{\mathcal{F} L})(\bar{y})=\left(\pi^{1}\right)^{-1}\left(\pi^{1}(\bar{y})\right)$, for all $\bar{y} \in J^{1} \pi$, and $\widetilde{\mathcal{F L}}^{-1}(\widetilde{\mathcal{F} \mathcal{L}})(\bar{y}) \subseteq \mathcal{F} \mathcal{L}^{-1}(\mathcal{F} \mathcal{L})(\bar{y}) \subseteq\left(\pi^{1}\right)^{-1}\left(\pi^{1}(\bar{y})\right)$, we obtain $\mathcal{F} \mathcal{L}^{-1}(\mathcal{F} \mathcal{L})(\bar{y})=$ $\widetilde{\mathcal{F} L}^{-1}(\widetilde{\mathcal{F} \mathcal{L}})(\bar{y})=\left(\pi^{1}\right)^{-1}\left(\pi^{1}(\bar{y})\right)$, and hence the fibers of $\mathcal{F} \mathcal{L}$ are connected submanifolds of $J^{1} \pi$. In conclusion, affine Lagrangian systems are almost regular.

Note that the manifold $\mathcal{P}$ can be identified with $E$, and the mapping $\mathcal{F} \mathcal{L}_{0}: J^{1} \pi \rightarrow \mathcal{P}$ can be identified with the mapping $\pi^{1}: J^{1} \pi \rightarrow E$. Hence, the $(m+1)$-form $\Omega_{h}^{0}=\left(\tilde{j}_{0} \circ \tilde{h}\right)^{*} \Omega$ (resp. the $m$-form $\left.\omega_{0}^{*}\right)$ on $\mathcal{P}$ can be identified with the $(m+1)$-form $-d \alpha$ (resp. $\left.\pi^{*}(\eta)\right)$ on $E$. Taking these identifications into account, the constrained Hamilton equations on $E$ are

$$
i\left(\mathcal{X}^{\mathcal{P}}\right)(d \alpha)=0, \quad i\left(\mathcal{X}^{\mathcal{P}}\right)\left(\pi^{*}(\eta)\right)=1 .
$$

Let $\nabla_{0}^{*}$ be a connection in the bundle $\tau_{0}^{1}: \mathcal{P} \rightarrow M$, and $\mathcal{Y}_{\eta}^{\nabla_{0}^{*}}=\bigwedge_{\mu=1}^{m}\left(\frac{\partial}{\partial x^{\mu}}+\Gamma_{\mu}^{A} \frac{\partial}{\partial y^{A}}\right)$ the corresponding $m$-vector field on $\mathcal{P}$ associated with $\nabla_{0}^{*}$ and $\eta$. A direct computation shows that

$$
\begin{gathered}
\left(\gamma_{h}\right)_{\eta}^{\nabla_{0}^{*}}=i\left(\mathcal{Y}_{\eta}^{\nabla_{0}^{*}}\right) \Omega_{h}^{0}=\left(-1^{m}\right)\left[\frac{\partial f_{A}^{\nu}}{\partial x^{\nu}}-\frac{\partial a}{\partial y^{A}}+\Gamma_{\nu}^{B}\left(\frac{\partial f_{A}^{\nu}}{\partial y^{B}}-\frac{\partial f_{B}^{\nu}}{\partial y^{A}}\right)\right]\left(d y^{A}-\Gamma_{\mu}^{A} d x^{\mu}\right), \\
\Omega_{h}^{\nabla_{0}^{*}}=\Gamma_{\nu}^{B}\left(\frac{\partial f_{B}^{\nu}}{\partial y^{A}}-\frac{\partial f_{A}^{\nu}}{\partial y^{B}}\right) d y^{A} \wedge d^{m} x-\frac{\partial f_{B}^{\mu}}{\partial y^{A}} d y^{A} \wedge d y^{B} \wedge d^{m-1} x_{\mu} .
\end{gathered}
$$

It is easy to show that $\Omega_{h}^{\nabla_{0}^{*}}$ is 1-nondegenerate if, and only if, the matrix $\left(f_{A B}^{\mu}\right)=\left(\frac{\partial f_{B}^{\mu}}{\partial y^{A}}-\frac{\partial f_{A}^{\mu}}{\partial y^{B}}\right)$ is regular, for every $\mu \in\{1, \ldots, m\}$. Then, the Hamiltonian constrained system (42) has solution. 
A $m$-vector field $\mathcal{X}^{\mathcal{P}}=\bigwedge_{\mu=1}^{m}\left(\frac{\partial}{\partial x^{\mu}}+F_{\mu}^{A} \frac{\partial}{\partial y^{A}}\right)$ is a solution to (42) on $\mathcal{P} \simeq E$ if, and only if,

$$
\left(\frac{\partial f_{A}^{\mu}}{\partial y^{B}}-\frac{\partial f_{B}^{\mu}}{\partial y^{A}}\right) F_{\mu}^{B}=\frac{\partial a}{\partial y^{A}}-\frac{\partial f_{A}^{\nu}}{\partial x^{\nu}}
$$

Note that there are $n$ equations and $m n$ variables, and that the rank of the matrix $\left(\frac{\partial f_{A}^{\mu}}{\partial y^{B}}-\frac{\partial f_{B}^{\mu}}{\partial y^{A}}\right)$ of type $n \times n m$ is maximum, that is, $n$. Thus, the set of solutions of the system is an affine space of dimension $n(m-1)$ (the solution is not unique if $m>1$ ).

With respect to the integrability of the solutions, a direct computation shows that a $m$-vector field $\mathcal{X}^{\mathcal{P}}$ solution to (42) is integrable if

$$
\frac{\partial F_{\nu}^{A}}{\partial x^{\mu}}-\frac{\partial F_{\mu}^{A}}{\partial x^{\nu}}+F_{\mu}^{B} \frac{\partial F_{\nu}^{A}}{\partial y^{B}}-F_{\nu}^{B} \frac{\partial F_{\mu}^{A}}{\partial y^{B}}=0, \text { for all } A \text { and } \mu, \nu .
$$

Otherwise, the integrability algorithm should be applied.

Taking into account the identification $\mathcal{P} \simeq E$, as $\Omega_{\mathcal{L}}=\mathcal{F} \mathcal{L}_{0}^{*}(-d \alpha)$, if $\mathcal{X}^{\mathcal{P}}$ is a solution to the constrained Hamiltonian equations on $\mathcal{P}$, then every locally decomposable $m$-vector field $\mathcal{X}^{J^{1} \pi}$ which projects via $\mathcal{F} \mathcal{L}_{0}$ onto $\mathcal{X}^{\mathcal{P}}$ is a solution to the equations

$$
i\left(\mathcal{X}^{J^{1} \pi}\right)\left(\Omega_{\mathcal{L}}\right)=0, \quad i\left(\mathcal{X}^{J^{1} \pi}\right) \omega=1 .
$$

Let $\Psi$ be the first-order jet field with respect to the fibration $\pi: E \rightarrow M$ associated to the Ehresmann connection defined by the $m$-vector field $\mathcal{X}^{\mathcal{P}}$. Then, the submanifold $S$ of $J^{1} \pi$ where a semi-holonomic $m$ vector field satisfying the Lagrangian equations exists is $\Psi(E)$. In fact, if $\mathcal{X}^{\mathcal{S}}=\left(\Lambda^{m} \mathrm{~T} \Psi\right) \circ \mathcal{X}^{\mathcal{P}}$ then $\mathcal{X}^{S}$ is an Euler-Lagrange $m$-vector field on $S$ for $\mathcal{L}$, that is, $\mathcal{X}^{S}$ is a locally decomposable $m$-vector field on $S$ and

$$
\left.\left(i\left(\mathcal{X}^{S}\right) \Omega_{\mathcal{L}}\right)\right|_{S}=0,\left.\quad\left(i\left(\mathcal{X}^{S}\right) \omega\right)\right|_{S}=1,\left.\quad\left(i\left(\mathcal{X}^{S}\right) \mathcal{V}\right)\right|_{S}=0
$$

If the matrix $\left(f_{A B}^{\mu}\right)$ is singular but there are no higher-order constraints, the previous results remain true. Otherwise, we will have to apply the premultisymplectic constraint algorithm. Suppose that we have obtained the final constraint submanifolds $N_{f}$ and $P_{f}$, with the submersion $\left.\left(\pi^{1}\right)\right|_{N_{f}}: N_{f} \rightarrow$ $P_{f}$. Let $\mathcal{X}^{P_{f}}$ be a $m$-vector field solution of the constrained Hamiltonian equations. We have that $\pi\left(P_{f}\right)$ is an open subset of $M$ and that $\pi_{f}=\pi_{\mid P_{f}}: P_{f} \rightarrow \pi\left(P_{f}\right) \subseteq M$ is a fibration. Moreover, $J^{1} \pi_{f}$ is a submanifold of $J^{1} \pi$ (see Appendix). Now, let $\Psi$ be the first-order jet field with respect to the fibration $\pi_{f}: P_{f} \rightarrow \pi\left(P_{f}\right)$ associated to the $m$-vector field $\mathcal{X}^{P_{f}}$. Then, the submanifold $S_{f}$ of $J^{1} \pi$ where an Euler-Lagrange $m$-vector field for $\mathcal{L}$ along $S_{f}$ exists is $\Psi\left(P_{f}\right)$, and $\mathcal{X}^{S_{f}}=\left(\Lambda^{m} T \Psi\right) \circ \mathcal{X}^{P_{f}}$ is such an Euler-Lagrange $m$-vector field (see Theorem 6).

Example: Let $\pi: \mathbb{R}^{4} \rightarrow \mathbb{R}^{2}$ be the configuration bundle, and $L=x^{2}\left(y^{1} v_{2}^{1}+y^{2} v_{2}^{2}\right)+y^{1} y^{2}$. In this case, $\alpha=y^{1} y^{2} d x^{1} \wedge d x^{2}-x^{2} y^{1} d y^{1} \wedge d x^{1}-x^{2} y^{2} d y^{2} \wedge d x^{1}$. If $\nabla$ is the trivial connection, $\mathcal{Y}_{\eta}^{\nabla}=\frac{\partial}{\partial x^{1}} \wedge \frac{\partial}{\partial x^{2}}$, then $\gamma_{\eta}^{\nabla}=\left(y^{1}-y^{2}\right)\left(d y^{1}-d y^{2}\right)$ and $\Omega_{\mathcal{L}}^{\nabla}=0$. A simple computation shows that, in this case, $b_{\Omega}^{\nabla}(\mathbf{h})=\left((\mathbf{h})_{\nabla}^{H}, 0\right)$. Therefore, the vector fields $Z_{i}$ in Theorem 5 are all the vertical vector fields in $\mathrm{V}\left(\bar{\pi}^{1}\right)$. Hence, the submanifold $N_{1}$ is characterized by the constraint $y^{1}-y^{2}=0$. In fact, every semi-holonomic 2-vector field in $N_{1}$ is an Euler-Lagrange 2-vector field for this problem. 


\section{Appendix: $m$-vector fields and Ehresmann connections in fibre bun- dles}

(See 10, 26, 30] for the proofs and other details about the results in this section).

Let $F$ be a $N$-dimensional differentiable manifold. Sections of $\Lambda^{m}(\mathrm{~T} F)$ are called multivector fields in $F$, or more precisely, $m$-vector fields in $F$ (they are contravariant skew-symmetric tensors of order $m$ in $F$ ). The space of $m$-vector fields is denoted by $\mathcal{V}^{m}(F) . \mathcal{X} \in \mathcal{V}^{m}(F)$ is locally decomposable if, for every $p \in F$, there exists an open neighbourhood $U_{p} \subset F$ and $Y_{1}, \ldots, Y_{m} \in$ $\mathfrak{X}\left(U_{p}\right)$ such that $\mathcal{X} \underset{U_{p}}{=} Y_{1} \wedge \ldots \wedge Y_{m}$. We denote by $\mathfrak{X}^{m}(F)$ the set of locally decomposable $m$-vector fields in $F$. Contraction of $m$-vector fields and tensor fields in $F$ is the usual one.

We can define an equivalence relation: if $\mathcal{X}, \mathcal{X}^{\prime} \in \mathfrak{X}^{m}(F)$ are non-vanishing $m$-vector fields, and $U \subseteq F$ is a connected open set, then $\mathcal{X} \stackrel{U}{\sim} \mathcal{X}^{\prime}$ if there exists a non-vanishing function $f \in \mathrm{C}^{\infty}(U)$ such that $\mathcal{X}^{\prime}=f \mathcal{X}$. Equivalence classes are denoted by $\{\mathcal{X}\}_{U}$. There is a one-to-one correspondence between the set of $m$-dimensional orientable distributions $D$ in $F$ and the set of the equivalence classes $\{\mathcal{X}\}_{F}$ of non-vanishing, locally decomposable $m$-vector fields in $F$. If $\mathcal{X} \in \mathfrak{X}^{m}(F)$ is nonvanishing and locally decomposable, the distribution associated with the class $\{\mathcal{X}\}_{U}$ is denoted $\mathcal{D}_{U}(\mathcal{X})$ (If $U=F$ we write $\mathcal{D}(\mathcal{X})$ ). A non-vanishing, locally decomposable $m$-vector field $\mathcal{X} \in$ $\mathfrak{X}^{m}(F)$ is said to be integrable if its associated distribution $\mathcal{D}_{U}(\mathcal{X})$ is integrable. Of course, if $\mathcal{X} \in \mathfrak{X}^{m}(F)$ is integrable, then so is every $m$-vector field in its equivalence class $\{\mathcal{X}\}$, and all of them have the same integral manifolds. Moreover, from Frobenius' theorem, a non-vanishing and locally decomposable $m$-vector field is integrable if, and only if, $\mathcal{D}(\mathcal{X})$ is involutive.

Now, let $\kappa: F \rightarrow M$ be a fibre bundle $(\operatorname{dim} M=m)$. We are concerned with the case where the integral manifolds of integrable $m$-vector fields in $F$ are sections of $\kappa$. Thus, $\mathcal{X} \in \mathfrak{X}^{m}(F)$ is said to be $\kappa$-transverse if, at every point $y \in F,\left(i(\mathcal{X})\left(\kappa^{*} \eta\right)\right)_{y} \neq 0$, for every $\eta \in \Omega^{m}(M)$ such that $\eta(\kappa(y)) \neq 0$. Then, if $\mathcal{X} \in \mathfrak{X}^{m}(F)$ is integrable, it is $\kappa$-transverse if, and only if, its integral manifolds are local sections of $\kappa: F \rightarrow M$. In this case, if $\phi: U \subset M \rightarrow F$ is a local section with $\phi(x)=y$ and $\phi(U)$ is the integral manifold of $\mathcal{X}$ through $y$, then $\mathrm{T}_{y}(\operatorname{Im} \phi)$ is $\mathcal{D}_{y}(\mathcal{X})$. Integral sections $\phi$ of $\mathcal{X}$ can be characterized by the condition

$$
\Lambda^{m} \mathrm{~T} \phi=f \mathcal{X} \circ \phi \circ \varrho_{M}
$$

where $\Lambda^{m} \mathrm{~T} \phi: \Lambda^{m} \mathrm{~T} M \rightarrow \Lambda^{m} \mathrm{~T} F$ is the natural lifting of $\phi, \varrho_{M}: \Lambda^{m} \mathrm{~T} M \rightarrow M$ is the natural projection, and $f \in \mathrm{C}^{\infty}(F)$ is a non-vanishing function (observe that this characterizes the entire class $\{\mathcal{X}\}$ of integrable $m$-vector fields).

Let $\nabla$ be an Ehresmann connection in the fibration $\kappa: F \rightarrow M$. As is known, it defines a horizontal subbundle $\mathrm{H}(\nabla) \subset \mathrm{T} F$, such that $\mathrm{T} F=\mathrm{H}(\nabla) \oplus \mathrm{V}(\kappa)$, where $\mathrm{V}(\kappa)$ is the $\kappa$-vertical subbundle. If $y \in F$, then $\mathrm{H}_{y}(\nabla)=\operatorname{Im} \nabla(y)$. Thus, we have the horizontal distribution associated with the connection $\nabla$. The connection $\nabla$ is said to be flat (respectively, orientable) if the horizontal distribution is completely integrable (respectively, orientable).

Classes of locally decomposable and $\kappa$-transverse $m$-vector fields $\{\mathcal{X}\} \subseteq \mathfrak{X}^{m}(F)$ are in one-toone correspondence with orientable Ehresmann connections $\nabla$ in $\kappa: F \rightarrow M$. This correspondence is given by the fact that the horizontal subbundle associated with $\nabla$ is $\mathcal{D}(\mathcal{X})$. Thus, classes of integrable locally decomposable and $\kappa$-transverse $m$-vector fields correspond to flat orientable Ehresmann connections. 
A connection $\nabla$ in the fibration $\kappa: F \rightarrow M$ induces a splitting $\mathrm{T}^{*} F=\mathrm{H}^{*}(\nabla) \oplus \mathrm{V}^{*}(\kappa)$, where

$$
\mathrm{H}_{y}^{*}(\nabla)=\mathrm{V}_{y}(\kappa)^{0}, \quad \mathrm{~V}_{y}^{*}(\kappa)=\mathrm{H}_{y}(\nabla)^{0} .
$$

Here, $\mathrm{V}_{y}(\kappa)^{0} \subset \mathrm{T}_{y}^{*} F$ (respectively, $\mathrm{H}_{y}(\nabla)^{0} \subset \mathrm{T}_{y}^{*} F$ ) denotes the annihilator of the subspace $\mathrm{V}_{y}(\kappa) \subset$ $\mathrm{T}_{y} F$ (respectively, $\mathrm{H}_{y}(\nabla) \subset \mathrm{T}_{y} F$ ). The splittings $\mathrm{T} F=\mathrm{H}(\nabla) \oplus \mathrm{V}(\kappa)$ and $\mathrm{T}^{*} F=\mathrm{H}^{*}(\nabla) \oplus \mathrm{V}^{*}(\kappa)$ may be extended to the tensor bundles

$$
\begin{gathered}
\Lambda^{l} \mathrm{~T} F=\bigoplus_{r, s=0, \ldots, l ; r+s=l}\left(\Lambda^{r} \mathrm{H}(\nabla) \oplus \Lambda^{s} \mathrm{~V}(\kappa)\right) \\
\Lambda^{k} \mathrm{~T}^{*} F=\bigoplus_{p, q=0, \ldots, k ; p+q=k}\left(\Lambda^{p} \mathrm{H}^{*}(\nabla) \oplus \Lambda^{q} \mathrm{~V}(\kappa)^{*}\right)
\end{gathered}
$$

Thus, for every $X \in \mathfrak{X}(F)$, we obtain that $i(X) \nabla \equiv X_{\nabla}^{H}$ is an horizontal vector field, that is, a section of $\mathrm{H}(\nabla) \rightarrow F . X_{\nabla}^{H}$ is the horizontal component of $X$, and we write $X=X_{\nabla}^{H}+X_{\nabla}^{V}$, where $X_{\nabla}^{V}=X-X_{\nabla}^{H}$ is a $\kappa$-vertical vector field. Moreover, if $\alpha \in \Omega^{1}(F)$, then we have that $i(\alpha) \nabla \equiv \alpha_{\nabla}^{H} \in \Omega^{1}(F)$ is an horizontal 1-form, that is, a section of $\mathrm{H}(\nabla)^{*} \rightarrow F$. $\alpha_{\nabla}^{H}$ is the horizontal component of $\alpha$, and we write $\alpha=\alpha_{\nabla}^{H}+\alpha_{\nabla}^{V}$, where $\alpha_{\nabla}^{V}=\alpha-\alpha_{\nabla}^{H}$ is a $\kappa$-vertical 1-form with respect to the connection $\nabla$, that is, it vanishes under the action of every horizontal vector field associated with the connection $\nabla$. Furthermore, if $X \in \mathfrak{X}(F)$ is a $\kappa$-vertical vector field, then $i(X) \alpha_{\nabla}^{H}=0$. In addition, if $\mathcal{X} \in \mathfrak{X}^{k}(F)$ and $\beta \in \Omega^{l}(F)$, the splittings (43) and (44) allow us to make the following decomposition

$$
\mathcal{X}=\sum_{r, s=0 ; r+s=k} \mathcal{X}_{\nabla}^{(r, s)}, \quad \beta=\sum_{p, q=0 ; p+q=l} \beta_{\nabla}^{(p, q)}
$$

where the superscripts $(i, j)$ denote the horizontal and vertical parts respectively, of the $k$-vector field $\mathcal{X}$ and the $l$-form $\beta$.

Finally, if $\nabla$ is an Ehresmann connection in the fibration $\kappa: F \rightarrow M$ and $y \in F$ then the map

$$
\Lambda^{k} \mathrm{~T}_{y} \kappa_{\mathrm{H}(\nabla)}: \Lambda^{k} \mathrm{H}_{y}(\nabla) \rightarrow \Lambda^{k} \mathrm{~T}_{\kappa(y)} M, \quad 1 \leq k \leq \operatorname{dim} M=m,
$$

is a linear isomorphism and the inverse morphism $\left(\Lambda^{m} \mathrm{~T}_{y} \kappa_{\mathrm{H}(\nabla)}\right)^{-1}: \Lambda^{m} \mathrm{~T}_{\kappa(y)} M \rightarrow \Lambda^{m} \mathrm{H}_{y}(\nabla)$ is just the horizontal lift at $y$ induced by $\nabla$. Denoting by $\Lambda^{m}\left(\kappa_{\mathrm{H}(\nabla)}\right)_{*}^{-1}$ the natural extension of this map to $m$-vector fields on $M$, one may consider $\Lambda^{m}\left(\kappa_{\mathrm{H}(\nabla)}\right)_{*}^{-1}(\mathcal{X})$, the horizontal lift of $\mathcal{X} \in \mathfrak{X}^{m}(M)$, as the $m$-vector field on $F$ given by

$$
\left[\Lambda^{m}\left(\kappa_{\mathrm{H}(\nabla)}\right)_{*}^{-1}(\mathcal{X})\right](y)=\left(\Lambda^{m} \mathrm{~T}_{y} \kappa_{\mathrm{H}(\nabla)}\right)^{-1}(\mathcal{X}(\kappa(y))), \quad \text { for every } y \in F .
$$

In particular, if $\mathcal{X}_{\eta}$ is the $m$-vector field on $M$ characterized by the condition

$$
\alpha_{1} \wedge \cdots \wedge \alpha_{m}=\mathcal{X}_{\eta}\left(\alpha_{1}, \ldots, \alpha_{m}\right) \eta \quad, \quad \text { for every } \alpha_{1}, \ldots, \alpha_{m} \in \Omega^{1}(M)
$$

one may define the $m$-vector field $\mathcal{Y}_{\eta}^{\nabla} \in \mathfrak{X}^{m}(F)$ by $\mathcal{Y}_{\eta}^{\nabla}=\Lambda^{m}\left(\kappa_{\mathrm{H}(\nabla)}\right)_{*}^{-1}\left(\mathcal{X}_{\eta}\right)$. Note that $\mathcal{Y}_{\eta}^{\nabla}$ is a locally decomposable and $\kappa$-transverse $m$-vector field on $F$, verifying that $i\left(\mathcal{Y}_{\eta}^{\nabla}\right) \omega=1$, and that the distribution $\mathcal{D}\left(\mathcal{Y}_{\eta}^{\nabla}\right)$ is just the horizontal distribution associated with the connection $\nabla$.

If $C$ is a submanifold of $F$, and $\mathcal{X}_{C}$ is a locally decomposable $m$-vector field on $C$ such that

$$
i\left(\mathcal{X}_{C}(y)\right) \omega(y)=1, \quad \text { for every } y \in C
$$

then $\left.\kappa\right|_{C} \equiv \kappa_{C}: C \rightarrow M$ is a submersion. In fact, if $y \in C$ and $\mathcal{X}_{C}(y)=X_{C}^{1}(y) \wedge \cdots \wedge X_{C}^{m}(y)$, with $X_{C}^{i}(y) \in \mathrm{T}_{y} C$, then

$$
\eta(\kappa(y))\left(\mathrm{T}_{y} \kappa_{C}\left(X_{C}^{1}(y)\right), \ldots, \mathrm{T}_{y} \kappa_{C}\left(X_{C}^{m}(y)\right)\right)=1
$$


This implies that $\left\{\mathrm{T}_{y} \kappa_{C}\left(X_{C}^{1}(y)\right), \ldots, \mathrm{T}_{y} \kappa_{C}\left(X_{C}^{m}(y)\right)\right\}$ is a basis of $\mathrm{T}_{\kappa(y)} M$, and thus, $\mathrm{T}_{y} \kappa_{C}: \mathrm{T}_{y} C \rightarrow$ $\mathrm{T}_{\kappa(y)} M$ is an epimorphism. Therefore, $\kappa(C)$ is an open subset of $M$ and $\kappa_{C}: C \rightarrow \kappa(C)$ is a fibre bundle. Consequently, $\mathcal{X}_{C}$ defines an oriented Ehresmann connection in the fibration $\kappa_{C}: C \rightarrow \kappa(C)$ which, in the terminology of [26, 30], is said to be an (oriented) Ehresmann connection in the fibration $\kappa: F \rightarrow M$ along the submanifold $C$. Note that the canonical inclusion $\iota: J^{1} \kappa_{C} \rightarrow J^{1} \kappa$ is an embedding and, thus, $J^{1} \kappa_{C}$ is a submanifold of $J^{1} \kappa$.

Remark 5 It is well-known [4] that there exists a one-to-one correspondence between Ehresmann connections in the fibration $\kappa: F \rightarrow M$ and first-order jet fields with respect to $\kappa$, that is, sections of the fibration $\kappa^{1}: J^{1} F \rightarrow F$. In fact, let $\nabla$ be a connection in the fibration $\kappa: F \rightarrow M$, (that is, an element of $\Gamma\left(E, \kappa^{*} T^{*} M\right) \otimes \Gamma(F, T F)$ ), such that $\nabla^{*} \alpha=\alpha$, for every $\kappa$-semibasic form $\left.\alpha \in \Omega^{1}(F)\right)$, and $\mathrm{H}(\nabla)$ the associated horizontal subbundle. If $\left(\mathrm{T}_{y} \kappa_{\mathrm{H}(\nabla)}\right)^{-1}$ denotes the horizontal lift at $y$; for every $y \in F$, let $\phi: M \rightarrow F$ be a section of $\kappa$ passing through $y$, such that

$$
\left.\mathrm{T}_{\kappa(y)} \phi=\mathrm{T}_{y} \kappa_{\mathrm{H}(\nabla)}\right)^{-1}: \mathrm{T}_{\kappa(y)} M \rightarrow \mathrm{H}_{y}(\nabla) \subset \mathrm{T}_{y} F
$$

then we define the map

$$
\begin{aligned}
& \psi^{\nabla}: F \rightarrow \quad J^{1} F \\
& y \mapsto \quad\left(j^{1} \phi\right)(\kappa(y))
\end{aligned}
$$

which is a section of the fibration $\kappa^{1}: J^{1} F \rightarrow F$. Conversely, given a section $\psi^{\nabla}: F \rightarrow J^{1} F$, for every $\bar{y} \in J^{1} F$ with $\kappa^{1}(\bar{y})=y$, and a representative $\phi: M \rightarrow F$ of $\bar{y}$, we define the horizontal subspace $\mathrm{H}_{y}(\nabla):=\operatorname{ImT}_{y} \phi$, and $\mathrm{H}(\nabla):=\cup_{y} \mathrm{H}_{y}(\nabla)$. Thus we have identified the fibre $J_{y}^{1} F=$ $\left(\kappa^{1}\right)^{-1}(y)$ with the set

$$
\left\{\mathbf{h}_{y} \in \mathrm{T}_{\kappa(y)}^{*} M \otimes \mathrm{T}_{y} F \mid \mathrm{T}_{y} \kappa \circ \mathbf{h}_{y}=I d\right\}
$$

In particular, if we have a connection or, what is equivalent, a class of $\kappa$-transverse, locally decomposable $m$-vector fields in the fibration $\kappa: F \rightarrow M$, along a submanifold $C$ of $F$, and a representative $\mathcal{X}_{C}$ of this class, then $\kappa(C)$ is an open subset of $M, \kappa_{C}=\left.\kappa\right|_{C}: C \rightarrow \kappa(C)$ is a fibration, and $\mathcal{X}_{C}$ may be seen as a section $\psi_{C}^{\nabla}$ of the fibration $\kappa_{C}^{1}: J^{1} \kappa_{C} \rightarrow C$. Thus, $\psi_{C}^{\nabla}(y)$ is identified with a linear map from $\mathrm{T}_{\kappa(y)} M$ onto $\mathrm{T}_{y} C$, that is, an element $\mathbf{h}_{y} \in \mathrm{T}_{\kappa(y)}^{*} M \otimes \mathrm{T}_{y} C$, and

$$
\left(\mathrm{T}_{y} \kappa_{C} \circ \psi_{C}\right)(y)=\left(\left.\mathrm{T}_{y} \kappa_{C}\right|_{\mathrm{T}_{y} C} \circ \psi_{C}^{\nabla}\right)(y)=I d, \quad \text { for every } y \in C .
$$

\section{Acknowledgments}

We acknowledge the financial support of Ministerio de Educación y Ciencia, projects BFM200203493, BFM2003-01319 and MTM2004-7832. We thank Mr. Jeff Palmer for his assistance in preparing the English version of the manuscript.

\section{References}

[1] C. Batlle, J. Gomis, J.M. Pons, N. Román-Roy, "Equivalence between the Lagrangian and Hamiltonian formalism for constrained systems", J. Math. Phys. 27(12) (1986) 2953-2962.

[2] E. Binz, J. Sniatycki, H. Fisher, The Geometry of Classical fields, North Holland, Amsterdam, 1988.

[3] J.F. Cariñena, M. Crampin, L.A. Ibort, "On the multisymplectic formalism for first order field theories", Diff. Geom. Appl. 1 (1991) 345-374. 
[4] J.F. Cariñena, J. Fernández-Nuñez, "Geometric theory of time-dependent singular Lagrangians", Fortschr. Phys. 41(6) (1993) 517-552.

[5] D. Chinea, M. De León, J.C. Marrero, "The constraint algorithm for time-dependent Lagrangians", J. Math. Phys. 7 (1994) 3410-3447.

[6] M. Crampin, Jet Bundle Techniques in Analytical Mechanics, Quaderni del Consiglio Nazionale delle Ricerche, Gruppo Nazionale de Fisica Matematica, Florence, 471995.

[7] P.A.M. Dirac, Lectures on Quantum Mechanics, (Belfer Graduate School of Science), New York, Yeshiva University, 1964.

[8] A. Echeverría-Enríquez, M.C. Muñoz-Lecanda, N. Román-Roy, "Non-standard connections in classical mechanics", J. Phys. A: Math. Gen., 28(12) (1995) 5553-5567.

[9] A. Echeverría-Enríquez, M.C. Muñoz-Lecanda, N. Román-Roy, "Geometry of Lagrangian first-order classical field theories". Forts. Phys. 44 (1996) 235-280.

[10] A. Echeverría-Enríquez, M.C. Muñoz-Lecanda, N. Román-Roy, "Multivector Fields and Connections. Setting Lagrangian Equations in Field Theories". J. Math. Phys. 39(9) (1998) 4578-4603.

[11] A. Echeverría-Enríquez, M.C. Muñoz-Lecanda, N. Román-Roy, "Multivector Field Formulation of Hamiltonian Field Theories: Equations and Symmetries", J. Phys. A: Math. Gen. 32 (1999) 8461-8484.

[12] A. Echeverría-Enríquez, M.C. Muñoz-Lecanda, N. Román-Roy, "Geometry of Multisymplectic Hamiltonian First-order Field Theories", J. Math. Phys. 41(11) (2000) 7402-7444.

[13] G. Giachetta, L. Mangiarotti, G. Sardanashvily, New Lagrangian and Hamiltonian Methods in Field Theory, World Scientific Pub. Co., Singapore (1997).

[14] G. Giachetta, L. Mangiarotti, G. Sardanashvily, "Differential Geometry of Time-Dependent Mechanics", dg-ga/9702020

[15] M.J. Gotay, J.M. Nester, G. Hinds, "Presymplectic manifolds and the Dirac-Bergmann theory of constraints", J. Math. Phys. 27 (1978) 2388-2399.

[16] M.J. Gotay, J.M. Nester, "Presymplectic Lagrangiany systems I: the constraint algorithm and the equivalence problem", Ann. Inst. H. Poincaré A 30 (1979) 129-142.

[17] M.J. Gotay, J.M. Nester, "Presymplectic Lagrangian systems II: the second order equation problem", Ann. Inst. H. Poincaré A 32 (1980) 1-13.

[18] X. Gràcia, J.M. Pons, "A generalized geometric framework for constrained systems", Diff. Geom. Appl. 2 (1992) 223-247.

[19] X. GrÀcia, R. Martín, "Time-dependent singular differential equations", Int. J. Geom. Methods Mod. Phys. (2005) (to appear).

[20] A.J. Hanson, T. Regge, C. Teitelboim, Constraint Hamiltonian Systems, Acad. Nazionale dei Lincei, Rome 1976.

[21] F. HÉLein, J. Kouneiner, "Finite dimensional Hamiltonian formalism for gauge and field theories", J. Math. Phys. 43(5) (2002) 2306-2347.

[22] L.A. Ibort, M. de León, J.C. Marrero, D. Martín de Diego, "Dirac brackets in constrained dynamics", Forschritte für Physik.47 5 (1999), 459-492.

[23] R.E. Kabala, F.E. Udwadia, Analytical Dynamics: A new Approach, Cambridge University Press, New York 1996.

[24] K. Kamimura, "Singular Lagrangians and constrained Hamiltonian systems, generalized canonical formalism", Nuovo Cim. B 69 (1982) 33-54. 
[25] I.V. Kanatchikov, "Canonical structure of Classical Field Theory in the polymomentum phase space", Rep. Math. Phys. 41(1) (1998) 49-90.

[26] M. de León, J. Marín-Solano, J.C. Marrero, "A Geometrical approach to Classical Field Theories: A constraint algorithm for singular theories", Proc. on New Developments in Differential geometry, L. Tamassi-J. Szenthe eds., Kluwer Acad. Press, (1996) 291-312.

[27] M. de León, J. Marín-Solano, J.C. Marrero, "The constraint algorithm in the jet formalism", Diff. Geom. Appl. 6 (1996) 275-300.

[28] M. de León, J. Marín-Solano, J.C. Marrero, M.C. Muñoz-Lecanda, N. Román-Roy, "Singular Lagrangian Systems on Jet bundles", Fortschr. Phys. 50(2) (2002) 105-169.

[29] M. de León, J.C. Marrero, D. Martín de Diego, "Time-dependent constrained Hamiltonian systems and Dirac brackets", J. Phys. A: Math. Gen. 29 (1996) 6843-6859.

[30] M. de León, J.C. Marrero, D. Martín De Diego, "A new geometric setting for Classical Field theories", Classical and Quantum Integrability (Warsaw, 2001), Banach Center Publ. 59, Polish Acad. Sci., Warsaw (2003) 189-209.

[31] L. Mangiarotti, G. Sardanashvily, "Gauge Mechanics", World Scientific, Singapore, 1998.

[32] L. Mangiarotti, G. Sardanashvily, "Connections in Classical and Quantum Field Theory", World Scientific, Singapore, 2000.

[33] L. Mangiarotti, G. Sardanashvily, "Constraints in the Hamiltonian Time-dependent Mechanics", J. Math. Phys. 41 (2000) 2858-2876.

[34] L. Mangiarotti, G. Sardanashvily, "Constraints in Polysymplectic (covariant) Hamiltonian formalism", math-ph/0008024 (2000).

[35] G. Marmo, G. Mendella, W.M. Tulcczyjew, "Constrained Hamiltonian systems as implicit differential equations", J. Phys. A 30(1) (1997) 277-293.

[36] E. Massa, S. Vignolo, "A new geometrical framework for time-dependent Hamiltonian Mechanics", Extracta Math. 18(1) (2003) 107-118.

[37] M.C. MuÑoz-LeCAndA, "Hamiltonian systems with constraints: a geometric approach". Int. J. Theor. Phys. 28 (11) (1989) 1405-1417.

[38] M.C. Muñoz-Lecanda, N. Román-Roy, "Lagrangian theory for presymplectic systems", Ann. Inst. H. Poincaré A 57(1) (1992) 27-45.

[39] G. Sardanashvily, Generalized Hamiltonian Formalism for Field Theory. Constraint Systems, World Scientific, Singapore (1995).

[40] G. Sardanashvily, "Hamiltonian Time-dependent mechanics", J. Math. Phys. 39(5) (1998) 27142729 .

[41] D.J. Saunders, The Geometry of Jet Bundles, London Math. Soc. Lect. Notes Ser. 142, Cambridge, Univ. Press, 1989.

[42] R. Skinner, R. Rusk, Generalized Hamiltonian dynamics I: Formulation on $T^{*} Q \otimes T Q "$, J. Math. Phys. 24 (1983) 2589-2594.

[43] S. Vignolo, "A new presymplectic framework for time-dependent Lagrangian systems: the constraint algorithm and the second-order differential equation problem", J. Phys. A: Math. Gen. 33 (2000) 5117-5135. 